\title{
Deletion and overexpression of the scaffolding protein IQGAP1 promotes HCC
}

Evan R Delgado ${ }^{1, *}$, Hanna L Erickson ${ }^{2, *}$, Junyan Tao ${ }^{1}$, Satdarshan P Monga ${ }^{1}$, Andrew W

Duncan $^{1, \#}$ and Sayeepriyadarshini Anakk ${ }^{2, \#}$

${ }^{1}$ Department of Pathology, McGowan Institute for Regenerative Medicine, Pittsburgh Liver

Research Center, University of Pittsburgh, Pittsburgh, PA. ${ }^{2}$ Department of Molecular and

Integrative Physiology, Cancer Center at Illinois, University of Illinois at Urbana-Champaign,

Urbana, IL 61801.

*denotes equal contribution and co-first authors, "denotes co-corresponding authors.

Full name, mailing address, phone and fax numbers, and email address of corresponding author

Sayeepriyadarshini Anakk

453 Medical Sciences Building

506 S Mathews Avenue

Urbana, IL 61801

Office: (217) 300-7905

Fax: (217) 244-5858

anakk@illinois.edu

Andrew W. Duncan

Bridgeside Point II, Suite 300

450 Technology Drive

Pittsburgh, PA 15219

Office: (412) 624-5302

Fax: (412) 624-5363

duncana@pitt.edu

Conflict of interest disclosure statement

There are no conflicts of interest.

\section{Word count}

6,494

\section{Number of figures and tables}

Figures: 7 main, 9 supplemental

Tables: 3 supplemental 


\begin{abstract}
IQ motif-containing GTPase-activating protein 1 (IQGAP1) is a ubiquitously expressed scaffolding protein that is overexpressed in a number of cancers, including liver cancer, and is associated with many pro-tumorigenic processes including cell proliferation, motility, and adhesion. IQGAP1 can integrate multiple signaling pathways and could be an effective antitumor target. Therefore, we examined the role for IQGAP1 in tumor initiation and promotion during liver carcinogenesis. Unexpectedly, we found that Iqgap $1^{-/-}$mice had a higher tumor burden than $\operatorname{lqgap} 1^{+/+}$and lqgap $1^{+/}$mice following DEN-induced liver carcinogenesis. Iqgap $1^{-/-}$ tumors as well as knocking down IQGAP1 in hepatocellular carcinoma (HCC) cell lines resulted in increased MET activation and cellular proliferation. On the other hand, we uncovered IQGAP1 overexpression accelerates HCC development by YAP activation and subsequent NUAK2 expression. We demonstrate that increasing IQGAP1 expression in vivo does not alter $\beta$-catenin or MET activation. Taken together, we identify that both loss and gain of function of IQGAP1 promotes HCC development by two separate mechanisms in the liver. These results demonstrate that adequate amount of IQGAP1 is necessary to maintain a quiescent status of liver.
\end{abstract}




\section{Introduction}

Liver cancer has a high mortality rate that is mainly attributed to lack of effective systemic therapies [1]. For hepatocellular carcinoma (HCC), the major form of primary liver cancer, a large portion of cases are diagnosed at advanced stages [2] and only two systemic therapies extend overall survival by a few months $[1,3]$. Both modalities are multi-kinase inhibitors where Sorafenib targets the serine-threonine kinases Raf-1 and B-Raf, vascular endothelial growth factor receptors (VEGFR) 1-3, and platelet-derived growth factor receptor (PDGFR) [1] and Lenvatinib targets VEGFR 1-3, fibroblast growth factor receptors (FGFR) 1-4, PDGFRa, stem cell factor receptor (KIT), and rearranged during transfection (RET) [3]. Many other kinase inhibitors have failed to improve on this survival benefit in phase III trials [4-9]. It is clear that a better understanding of liver tumor biology is required to improve therapeutic strategies for HCC.

IQ motif-containing GTPase-activating protein 1 (IQGAP1) is a pleiotropic, multi-domain scaffolding protein that is overexpressed in many types of human cancer [10], including $60-80 \%$ of HCCs [11-14], and this overexpression is associated with worse clinical outcomes [13]. IQGAP1 interacts with pro-tumorigenic processes, including kinase signaling, cell proliferation, motility, and adhesion [10]. Furthermore, in vivo studies demonstrate that increased IQGAP1 expression can promote tumor growth, indicating that IQGAP1 could be an effective molecular target for HCC [15-17]. Yet, other studies revealed that deletion of IQGAP1 in cancer cells and/or stromal cells can also enhance tumorigenesis by modulating transforming growth factor (TGF) signaling [18, 19] and adherens junction stability [20].

To understand these contradictory studies, in this paper we carefully investigated the role for IQGAP1 in hepatic tumorigenesis by directly comparing IQGAP1-knockout (lqgap1//) and IQGAP1-overexpression mouse models of HCC. Using the diethylnitrosamine (DEN) model of liver cancer, we show that deletion of both copies of lqgap1 increases the incidence and multiplicity of liver tumors. Iqgap $1^{-/}$tumors exhibited elevated levels of the tyrosine kinase 
receptor MET, and this could directly contribute to increased proliferation. Further, we show that overexpression of IQGAP1 enhances rapid onset and progression of HCC. YAP1 activation driven by an increase in NUAK2 kinase expression contribute to this model. Thus, we show that both deletion and overexpression of IQGAP1 can promote the development of liver tumors in mice using two distinct molecular mechanisms. Our findings underscore that molecular expression of liver tumors should be considered when developing new therapies for HCC.

\section{Results}

\section{Deletion of IQGAP1 promotes DEN-induced tumorigenesis}

To study whether IQGAP1 is required for hepatic tumorigenesis, we used the DEN model of liver cancer, a gold standard for chemical carcinogenesis that effectively mimics the prolonged development of HCC in humans following a previously published protocol [21]. We treated male and female lqgap $1^{+/+}$, Iqgap $1^{+/-}$, and $\operatorname{lqgap} 1^{-/-}$mice with $5 \mathrm{mg} / \mathrm{kg}$ DEN via intraperitoneal injection at 12-15 days of age and assessed tumor burden 20- and 50-weeks post-treatment. Visible tumors were observed in $0 / 9$ lqgap $1^{+/+}, 1 / 3$ lqgap $1^{+/}$, and $0 / 3$ lqgap $1^{-/-}$ female mice at the 50 -week time point, which is consistent with previous reports that females are protected from developing HCC $[22,23]$.

Because female mice are protected from developing HCC, we performed all further analysis on male mice only. In the male mice, no macroscopic nodules were observed at 20 weeks (Supplemental Figure 1A), but microscopic lesions were observed in 3/9 Iqgap $1^{+/+}$ (33.3\%), 3/10 Iqgap $1^{+/-}(30 \%)$, and 3/5 Iqgap $1^{-/-}(60 \%)$ livers at that time point (Supplemental Figure 1B). Macroscopic nodules were observed at 50 weeks post DEN treatment in 9/13 Iqgap $1^{+/+}(69.2 \%), 11 / 24$ lqgap $1^{+-}(45.8 \%)$, and 14/16 lqgap1/- $(87.5 \%)$ mice (Figure $\left.1 \mathrm{~A}-\mathrm{B}\right)$. We found higher incidence (Figure 1B) and multiplicity (Figure 1C) of liver tumors in lqgap $1^{-/-}$ compared to $\operatorname{lqgap} 1^{+/-}$mice, indicating that total loss of lqgap1 promotes tumorigenesis. However, lqgap1-deletion did not affect maximum tumor size (Figure 1D). When we segregate a 
subset of mice with LW/BW $>7 \%$ (Figure 1E), we found that lqgap $1^{-/-}$livers were significantly larger than Iqgap $1^{+/+}$and Iqgap $1^{+/-}$livers. These data suggest that the tumor burden is higher in lqgap $1^{-/}$animals.

We previously showed that lqgap $1^{-/}$mice have lower gonadal adipose tissue weight [24]. Since body composition can both affect and be affected by tumor burden, we asked whether the increased tumor burden in Iqgap $1^{-/-}$animals corresponded to differences in body composition. Body weight was approximately $14 \%$ lower in Iqgap $1^{-/}$animals $(31.2 \mathrm{~g})$ than both Iqgap $1^{+/+}(36.4 \mathrm{~g})$ and lqgap1 ${ }^{+/-}(37.0 \mathrm{~g})$ mice at 50 weeks post-DEN treatment (Supplemental Figure $2 \mathrm{~A}$ ). The same trend was observed as early as 10 weeks of age, indicating that the effect of lqgap1-deletion on body weight occurred prior to tumorigenesis (Supplemental Figure 2B). At this time point, no difference in white adipose tissue (WAT) or quadriceps muscle weight was observed (Supplemental Figure 2C-D). Levels of serum injury markers alanine aminotransferase (ALT), aspartate aminotransferase (AST), and total bilirubin were elevated but not different between groups (Supplemental Figure 2E-G). This suggests that the increased tumor burden in lqgap $1^{-/}$animals is likely not due to differences in body composition or increased liver injury.

We next confirmed that deletion of Iqgap1 did not result in compensatory expression changes in homologs lqgap2 and Iqgap3. Of the three isoforms, IQGAP1 has the widest tissue distribution and is more frequently altered in cancer [25]. As expected, lqgap1 was induced in $\operatorname{lqgap} 1^{+/+}$tumors relative to the surrounding healthy liver, and the $\operatorname{lqgap} 1^{+/-}$mice exhibited an approximately $50 \%$ reduction in lqgap1 expression compared to controls (Supplemental Figure 3A). Iqgap2 is more highly expressed in the control liver $\left(\right.$ Iqgap $1^{+/+}$liver $\left.\mathrm{Cq}=18-19\right)$ than Iqgap1 $\left(\right.$ lqgap1 $1^{+/+}$liver $\left.\mathrm{Cq}=23-24\right)$ but is decreased in tumor tissue (Supplemental Figure 3B). Whereas lqgap3 expression is low in the quiescent livers (Iqgap $1^{+/+}$liver $\left.\mathrm{Cq}=30-31\right)$, it is observed in proliferating cells [26, 27] and dramatically induced in tumor tissue (Supplemental 
Figure 3C). Notably, expression pattern of neither lqgap2 nor lqgap3 was altered by lqgap1deletion.

\section{Highly proliferative Iqgap $1^{-/}$tumors are characterized by elevated GS or activated ERK}

The incidence and multiplicity of tumors were higher in lqgap $1^{-/-}$mice, so we asked whether the tumors differed in proliferation or pathology. The DEN model generates both hepatocellular adenoma (HCA), a benign tumor, and hepatocellular carcinoma (HCC), a malignant tumor [21]. These can be histologically differentiated as HCC has a thick trabecular structure, while HCA has cells arranged in thin trabeculae or in cords similar to normal hepatocytes with no vasculature. Since some of the livers had multiple tumors, we characterized each tumor separately. Both tumor types were observed in all three genotypes and some livers had both HCA and HCC tumors. (Figure 1F). HCA nodules were found in 11/23 lqgap $1^{+/+}$ (47.8\%), 9/18 lqgap 1// (50.0\%), and 13/28 lqgap 1/- $(46.4 \%)$ tumors while HCC nodules were found in 12/23 lqgap $1^{+/+}(52.1 \%), 9 / 18$ lqgap $^{+/-}(50 \%)$, and 15/28 lqgap $1^{-/-}(53.6 \%)$ tumors.

We next asked if there were any fundamental differences in the molecular characteristics of these tumors. Liver cancer can be divided into at least six molecular subtypes (G1-G6) depending on their gene expression pattern (Supplemental Table 1) [28]. We analyzed markers of proliferation (Supplemental Figure 3D-E), lipogenesis (Supplemental Figure 3F), angiogenesis (Supplemental Figure 3G), inflammation (Supplemental Figure 3H), gluconeogenesis (Supplemental Figure 3I), and $\beta$-catenin activity (Supplemental Figure 3J) that have been previously identified to correspond to these distinct molecular subtypes of human HCC $[28,29]$. We found no difference in gene expression pattern between groups. The expression changes of 5/7 genes (Rrm2, Tgfbr1, Fasn, Pepck, Angpt2) aligned with G3 tumors, which are characterized by chromosomal instability, cell cycle activation, and TP53 mutations [28]. 
DEN promotes hepatocellular carcinogenesis by inducing covalent DNA adducts in hepatocytes that can increase the mutation rate and thus the risk of uncontrolled growth. There are 4 codons known to be frequently altered in DEN-induced tumors (BRAF K584, BRAF V637, EGFR F254, and HRAS Q61) [30, 31], and we assessed whether the prevalence of these DNA mutations varied among the groups as a marker for overall mutation rate. Of these, only BRAFV637 showed considerable percent mutation (Supplemental figure 4A-D). However, there was no difference in the amount mutated between groups. Furthermore, we assessed the initial proliferative response to DEN treatment at 24 hours after injection since an impaired response to injury can drive tumor growth. We found no difference in proliferation rate between groups (Supplemental figure 4E-F). Together, these data show that Iqgap1 deletion does not affect mutation rate or the initial proliferative response to DEN.

DEN-induced tumors are frequently driven by mutations in Hras and, to a lesser extent, mutations in Ctnnb1 [32, 33]. We examined whether deletion of IQGAP1 altered the activation of these pathways by performing immunohistochemistry for phosphorylated ERK (P-ERK) and glutamine synthetase (GS). We divided tumors into four categories: GS and P-ERK negative, PERK positive, GS positive, and both P-ERK and GS positive (Figure 2A). IQGAP1 deletion did not affect the zonal expression pattern of GS in liver tissue. Hras and Ctnnb1 mutations are typically mutually exclusive [34], which is consistent with our finding that a low number of tumors were both P-ERK and GS positive. HCAs were primarily P-ERK positive, with 9/11 Iqgap $1^{+/+}$ (81.2\%), 9/9 lqgap $1^{+-}(100.0 \%)$, and 12/13 lqgap 1/- $(92.3 \%)$ tumors shows positive staining (Figure 2B). On the other hand, a substantial proportion of HCCs had increased GS-positive staining (4/12 lqgap $1^{+/+}(33.3 \%), 2 / 9 \operatorname{lqgap1}^{+/-}(22.2 \%)$, and 7/15 lqgap $\left.1^{-/-}(46.7 \%)\right)$ (Figure 2C).

Then we investigated if the increased tumor burden is associated with increased proliferation. Ki-67, a marker of non-quiescent cells, staining revealed low levels of positive cells in healthy liver tissue from tumor-bearing mice, regardless of genotype (Figure 2D-E). However, there was a stark increase in $\mathrm{Ki}-67$ positive cells in tumors from lqgap $1^{-/}$mice compared to 
Iqgap $1^{+/+}$mice (Figure 2D-E). We checked markers of epithelial-mesenchymal transition in these tumors and found no significant difference in MMP2, E-cadherin, N-cadherin, or CDC42 protein expression between groups (Supplemental Figure 5). This indicates that lqgap1-deletion does not alter the molecular characteristics of the tumors, but it does increase their proliferation.

\section{Loss of IQGAP1 results in dysregulated MET expression and activation}

To identify the mechanism underlying tumorigenesis in lqgap $1^{-/} \mathrm{DEN}$-mediated HCC model, we first examined the Wnt pathway. At the RNA level, Ctnnb1 and its canonical target genes Birc5, and to a lesser extent Axin2 and Lect2 were induced in tumor tissue, regardless of genotype (Supplemental Figure 6A-D). Further, knock down of IQGAP1 in HepG2 cells, Huh7, and Snu-449 cells revealed no change in $\beta$-catenin activity, as determined by TOPFlash luciferase reporter assay (Supplemental figure 6E-F). Also, DEN carcinogenesis resulted in GS positive nodules across all cohorts, suggesting that lqgap1 loss does not exacerbate Wnt signaling.

Since DEN-induced Iqgap $1^{-/-}$tumors display either activated P-ERK or GS expression, we investigated tyrosine kinase receptor MET, which can activate both Ras and $\beta$-catenin signaling pathways [35-38]. We measured MET expression and activation, marked by the phosphorylation of tyrosine residues 1234/1235 of MET (Y1234/1235) [38]. Although Y1234/1235 phospho-MET (P-MET) levels remained unchanged between the groups, total MET levels were elevated in Iqgap $1^{-/-}$tissues after DEN (Figure 3A). Phosphorylated AKT-1 (P-AKT1) $S 473$, a target downstream of MET signaling, also trended higher in lqgap $1^{-/}$tissues, which is consistent with a pattern of MET induction (Figure 3A). Next, we examined if IQGAP1 knock down could directly regulate MET signaling in vitro using HepG2 and Snu449 cells. Knock down of IQGAP1 in cell lines was sufficient to increase MET expression (Figure 3B). Further, upon HGF stimulation IQGAP1 knock down cells showed increased phosphorylation of MET, AKT-1 and S6, suggesting that loss of IQGAP1 renders cells highly sensitive to MET pathway 
activation (Figure 3B). In addition, treatment of cells with the MET small molecule inhibitor EMD1214063 blocks MET, AKT-1 and S6 phosphorylation after IQGAP1 loss and HGF stimulation (Figure 3B). MET protein and RNA expression were also increased in HepG2 cells treated with shRNA for IQGAP1 (Figure 3C-D), but expression of $\beta$-catenin or its target Axin1 expression were not affected (Figure 3D). To determine whether the increased MET expression correlated with increased proliferation, we also checked the expression of CCND1, which was 2fold higher with IQGAP1 knock down (Figure 3D). We can also block elevated CCND1 following IQGAP1 loss using EMD1214063 (Figure 3E). Together, the data indicate that loss of IQGAP1 results in increased MET signaling and proliferation, which could facilitate HCC oncogenesis.

\section{IQGAP1 overexpression exacerbates HCC carcinogenesis in the $\beta$-catenin/MET model}

Since IQGAP1 expression is enhanced in the majority of HCC cases [11, 12], we asked whether overexpression of IQGAP1 was sufficient to promote tumor growth. To do this, we turned to a well-established HCC model that uses hydrodynamic tail vein injection (HDTVI) with the Sleeping Beauty (SB) transposase (referred hereafter as the "transposon system"). Briefly, the transposon system can induce HCC in wild-type (WT) adult mice by the forced expression of human activated $(S 45 Y) \beta$-catenin $+M E T(B+M)$ [39]. The S45Y mutation in $\beta$-catenin results in reduced degradation, enhanced nuclear translocation and activation of canonical Wnt signaling; the MET construct increases MET expression and downstream signaling events [39]. Simultaneous expression of $B+M$ induces microscopic lesions visible by 2 weeks and macroscopic HCC within 6-9 weeks, which are 69\% genetically similar to human HCC [39].

Using the transposon system, we overexpressed epitope tagged $\mathrm{B}+\mathrm{M}$ with or without simultaneous expression of epitope tagged human IQGAP1 $(B+M+I)$ in WT mice and harvested livers after 4 or 8.5 weeks (Figure $4 \mathrm{~A}$ ). As early as 4 weeks, tagged $\beta$-catenin and MET were expressed in liver lysates in $\mathrm{B}+\mathrm{M}$ and $\mathrm{B}+\mathrm{M}+\mathrm{I}$ groups, compared to non-treated (NT) controls, and tagged IQGAP1 was expressed only in the $\mathrm{B}+\mathrm{M}+\mathrm{I}$ group (Figure 4B). We also checked 
expression of Iqgap family members and found that Iqgap2 was unchanged while lqgap3 was elevated in both $\mathrm{B}+\mathrm{M}$ and $\mathrm{B}+\mathrm{M}+\mathrm{I}$ livers compared to NT controls (Supplemental Figure 7A-B). Upregulation of Iqgap3 in tumor tissues was also seen in DEN-induced tumors (Supplemental Figure $3 \mathrm{C}$ ), again suggesting that lqgap3 induction marks cell proliferation.

Macroscopic disease was not evident after 4 weeks, but at 8.5 weeks, there were visible macroscopic tumor nodules in both the $\mathrm{B}+\mathrm{M}$ and $\mathrm{B}+\mathrm{M}+\mathrm{I}$ groups (Figure $4 \mathrm{C}$ ). The LW/BW ratio (Figure 4D) was equivalent after 4 weeks but was 2 -fold higher in the $\mathrm{B}+\mathrm{M}+\mathrm{I}$ group at 8.5 weeks compared to controls. Alpha-fetoprotein, a marker of highly aggressive HCC [40-42], was highest in the $\mathrm{B}+\mathrm{M}+\mathrm{I}$ group at 8.5 weeks (Figure 4E). Microscopic tumor nodules were present at each of the time points (Figure 4F). Tumors in $\mathrm{B}+\mathrm{M}$ and $\mathrm{B}+\mathrm{M}+\mathrm{I}$ groups showed typical $\mathrm{HCC}$ morphology, visible by H\&E staining, which contrasts with the mix of tumor types seen in the DEN model (Figure 1F). As expected for tumors induced by activated $\beta$-catenin, HCC nodules were enriched for GS expression in both groups and at all time points. In addition, we measured the expression of genes that differentiate between G1-G6 molecular subtypes, as described in Supplemental Table 1, and found changes in 4/7 genes (Glul, Tgfbr1, Fasn, and Crp) in B+M and $\mathrm{B}+\mathrm{M}+\mathrm{I}$ tissues are representative of $\mathrm{G} 5 / \mathrm{G} 6$ tumors (Supplemental Figure $7 \mathrm{C}-\mathrm{I}$ ). Although Crp expression was lower in $\mathrm{B}+\mathrm{M}+\mathrm{I}$ tissues compared to $\mathrm{B}+\mathrm{M}$, we largely did not find differences in expression patterns between $B+M$ and $B+M+I$ samples. $H C C$ tumors with activating CTNNB1 mutations typically fall within the G5/G6 molecular subtypes, which are characterized by low cell proliferation, chromosomal stability, and a lack of inflammatory infiltrates $[28,29]$. Notably, HCC nodules at 8.5 weeks were advanced and highly necrotic (Figure 4F). Thus, IQGAP1 overexpression in the B+M tumor model accelerates HCC tumor expansion.

\section{Overexpression of IQGAP1 does not accelerate HCC formation via enhanced MET or}

\section{Wnt/ $\beta$-catenin signaling}


We sought to determine the mechanism by which IQGAP1 overexpression accelerates HCC formation in the transposon model. Deletion of IQGAP1 is associated with increased MET expression and activation (Figure 3), so we asked if IQGAP1 overexpression in vivo also impacts MET. MET is known to activate pro-tumorigenic pathways including MEK/ERK, $\mathrm{PI}$ IK/AKT/mTOR, and others, resulting in increased protein phosphorylation [38]. Therefore, we assessed $\mathrm{P}-\mathrm{MET} \mathrm{Y} 1234 / 1235$ in $\mathrm{B}+\mathrm{M}$ and $\mathrm{B}+\mathrm{M}+\mathrm{I}$ livers (Supplemental Figure 8). Total MET expression was equivalent between groups, and phosphorylated MET at Y1234/1235 was 2-fold higher in $\mathrm{B}+\mathrm{M}+\mathrm{I}$ (Supplemental Figure 8). However, downstream targets of MET activation (AKT-1, mTOR, and STAT3) were unchanged (Supplemental Figure 8), suggesting that the MET pathway is not the driving mechanism of HCC in this model.

Multiple studies have shown that IQGAP1 enhances Wnt/ $\beta$-catenin signaling in vitro [14, 43]. To verify this, we overexpressed IQGAP1 in cell culture (Figure 5A) and found 2 to 6 -fold increase in Wnt/ $\beta$-catenin activity upon IQGAP1 overexpression as measured by the TOPFlash reporter assay (Figure 5B). This induction was comparable to reporter activation achieved by an activated form of $\beta$-catenin alone. Next, we tested whether co-expression of IQGAP1 and $\beta$ catenin could increase reporter activity in an additive or synergistic manner. However, simultaneous expression of IQGAP1 and $\beta$-catenin did not change to $\beta$-catenin activity when compared to $\beta$-catenin alone (Figure 5C). Collectively, the data demonstrate that IQGAP1 overexpression can increase $\beta$-catenin activity in vitro, but this mechanism is more complex since overexpressing IQGAP1 and $\beta$-catenin together does not further enhance Wnt/ $\beta$-catenin signaling.

IQGAP1 is reported to assist translocation of $\beta$-catenin from the cytoplasm to the nucleus in vitro, resulting in enhanced Wnt/ $\beta$-catenin signaling [14]. Therefore, to directly test if IQGAP1 enhances $\beta$-catenin transcriptional activity in vivo, we investigated changes in $\beta$ catenin subcellular localization after IQGAP1 overexpression by isolating cytoplasmic and nuclear fractions from whole liver samples of $B+M$ or $B+M+I$ mice. As expected, IQGAP1 was 
elevated in the cytosol of $\mathrm{B}+\mathrm{M}+\mathrm{I}$ livers, compared to $\mathrm{B}+\mathrm{M}$ (Figure $5 \mathrm{D})$. This correlated with a striking 3-fold increase in cytosolic $\beta$-catenin (Figure 5D). In the nuclear fraction, a high level of IQGAP1 was found in $\mathrm{B}+\mathrm{M}+\mathrm{I}$ livers, demonstrating IQGAP1 translocation into the nucleus. Similarly, $\beta$-catenin levels showed modest but significant increase in the nucleus, consistent with previous findings (Figure 5D).

Since we and others have found increased IQGAP1 expression enhances $\beta$-catenin's transcriptional activity in vitro (Figures 5B-C) [14, 43], we investigated if IQGAP1-mediated tumor promotion increases Wnt/ $\beta$-catenin signaling in vivo by examining the expression of several canonical Wnt/ $\beta$-catenin target genes. First, we checked $\beta$-catenin mRNA expression and, similar to the protein expression reported in Figure $4 B$, found no difference between $B+M$ and $\mathrm{B}+\mathrm{M}+\mathrm{I}$ groups (Figure $5 \mathrm{E}$ ). Next, after 4 weeks there was no significant difference in expression of canonical $\beta$-catenin targets Birc5, Lect2, Ccdn1 or Axin2 between B+M and $\mathrm{B}+\mathrm{M}+\mathrm{I}$ groups (Figure 5E). However, after 8.5 weeks, expression of target genes was highly variable. For example, Birc5 was 2-fold higher in $\mathrm{B}+\mathrm{M}+\mathrm{l}$ livers compared to $\mathrm{B}+\mathrm{M}$ livers; Lect2 and Glul were significantly induced in both $\mathrm{B}+\mathrm{M}+\mathrm{I}$ and $\mathrm{B}+\mathrm{M}$ livers; $\mathrm{Ccdn1}$ expression was induced in the $\mathrm{B}+\mathrm{M}$ condition but not in $\mathrm{B}+\mathrm{M}+\mathrm{l}$ livers; and Axin2 was unchanged. Thus, since Birc5 expression is not exclusively controlled by Wnt/3-catenin signaling, and mRNA expression of Ctnnb1, Lect2, Ccdn1, Axin2 and Glul (Supplemental Figure 6I) are similar between B+M and $\mathrm{B}+\mathrm{M}+\mathrm{I}$ groups, we concluded that IQGAP1 overexpression has a limited impact on $\mathrm{Wnt} / \beta-$ catenin signaling in vivo.

IQGAP1 is known to regulate the physical interaction between $\beta$-catenin and $\mathrm{E}$-Cadherin at the cell membrane in vitro $[44,45]$. We, therefore, investigated if IQGAP1 overexpression enhances $\beta$-catenin-E-Cadherin complexes in vivo. Using whole liver lysates from $\mathrm{B}+\mathrm{M}$ or $\mathrm{B}+\mathrm{M}+\mathrm{I}$ mice, we co-immunoprecipitated $\beta$-catenin and probed for $\mathrm{E}$-Cadherin and found IQGAP1 overexpression enhances $\beta$-catenin-E-Cadherin interactions (Figure 5F). We also 
stained $B+M$ and $B+M+I$ tissues for tagged $\beta$-catenin, MET and IQGAP1 and found that $\beta$ catenin and IQGAP1 co-localize at the cell membrane in $\mathrm{B}+\mathrm{M}+\mathrm{I}$ tissues (Figure $5 \mathrm{G}$ ). These data consistently demonstrate that even though the $\mathrm{B}+\mathrm{M}+\mathrm{I}$ model shows increased tumor burden compared to the $\mathrm{B}+\mathrm{M}$ model, enhanced $\mathrm{Wnt} / \beta$-catenin signaling via IQGAP1 overexpression in vivo is not causing this result.

\section{IQGAP1 overexpression drives HCC via Hippo/YAP signaling}

Our data indicate IQGAP1 does not enhance Wnt/ß-catenin or MET signaling in the $\mathrm{B}+\mathrm{M}$ model. Since we found elevated mRNA expression of Birc5 and Glul (Figure 5E), which are also known targets of Hippo pathway [46, 47], we investigated its role in promoting tumorigenesis in the $\mathrm{B}+\mathrm{M}+\mathrm{I}$ model. Activation of Hippo kinases results in cytoplasmic retention of Yes-associated protein1 (YAP1) and thus inhibiting cellular proliferation. IQGAP1 has been shown to regulate YAP1 levels and activity $[48,49]$. Therefore, we examined the cytoplasmic to nuclear translocation of YAP1 when IQGAP1 is overexpressed in the B+M model. We found that nuclear YAP1 (2.5-fold) was increased in the $B+M+I$ samples, compared to $B+M$ (Figure 6A). Next, we examined the mRNA expression of Yap1 and its target genes (Amot/2, Ccn1, Ccn2 and Jag1) and found that their expression largely remained unchanged between $B+M$ and $\mathrm{B}+\mathrm{M}+\mathrm{I}$ groups except for $\mathrm{Ccn} 2$ (Figure 6B). This data is consistent with recent findings that reveal Amot/2, Ccn1, Ccn2 and Jag1 have minimal functional role in HCC pathogenesis in vivo [50-55] even though they are important in tracking hepatoblastoma progression [56-59].

More recently, YAP1-driven HCC tumorigenesis was shown to be mediated by NUAK2 kinase, which was also shown to positively regulate YAP1 activity in a feed forward manner [60, 61]. Interestingly, we found Nuak2 expression is maintained when IQGAP1 is overexpressed in the $\mathrm{B}+\mathrm{M}$ model at the 4-week time-point compared to $\mathrm{B}+\mathrm{M}$ alone where Nuak2 is derepressed (Figure 6C). However, this regulation is not observed at the 8.5-week time-point (Figure 6C), which suggests that IQGAP1-YAP1 regulation may be lost in late stage of tumorigenesis. 
Consistently, elevated YAP1 activity is known as an early oncogenic event in HCC [62] and data from Figure 6A and $\mathrm{C}$ suggest that IQGAP1 may stabilize YAP1 activity by promoting NUAK2 expression at early stages of HCC oncogenesis. To further understand the clinical implications of the IQGAP1-NUAK2 axis in HCCs, using The Cancer Genome Atlas (TCGA), we identified that $35 \%$ of patients with IQGAP1 ${ }^{\text {High }}$ mRNA expression also had NUAK2 $2^{\text {High }}$ expression (Figure $6 \mathrm{D}, \mathrm{n}=371)$. Patients with $/ Q G A P 1^{\text {High }} / N U A K 2^{\text {High }}$ expression exhibited a poor overall survival trend (Figure 6E) and their transcriptomic signature revealed activation of multiple pro-growth and pro-survival signaling pathways compared to other HCC patients (Supplemental Figure 9). Taken together, our data indicate that IQGAP1 overexpression in tumors exacerbates Hippo/YAP signaling via enhanced NUAK2 expression, which may be a druggable mechanism for a specific subset of HCC patients.

\section{Discussion}

Our results show that both loss and gain of IQGAP1, a scaffold protein, can promote HCC in the murine liver (Figure 7). Consistent with our finding, IQGAP1 is frequently induced and is associated with a worse prognosis in human $\mathrm{HCC}[11-14,63]$. Intriguingly, we found that IQGAP1-deletion also increased tumor burden. We reconcile these results and postulate that IQGAP1 has a bimodal effect on promoting hepatic tumorigenesis.

IQGAP1 loss does not predispose livers to develop differential subsets of liver cancer (e.g., HCA versus HCC) (Figure 1F) nor cause discrete molecular characteristics (Supplemental Figure 3). However, Iqgap1-deficient tumor lesions proliferate and rapidly develop liver cancer with increased severity (Figure 2D-E). We found that canonical Wnt/ $\beta$-catenin signaling was unaltered in lqgap $1^{-/}$livers (Supplemental Figure 6), despite previous studies showing that IQGAP1 facilitates $\beta$-catenin's nuclear translocation and activity $[14,43,64]$. Why $\beta$-catenin signaling does not change in the absence of IQGAP1 warrants future investigation. We found in vitro knock down of IQGAP1 increased CCND1 expression and MET expression in HepG2 cells 
(Figure 3B-D). MET expression was also induced in lqgap $1^{-/}$liver tumors suggesting that this increase may be compensatory to the loss of IQGAP1 expression, or IQGAP1 may regulate turnover of the MET protein.

On the other hand, we demonstrate that increased expression of IQGAP1 is sufficient to increase tumor burden and exacerbates $\mathrm{HCC}$ development in the $\mathrm{B}+\mathrm{M}$ model (Figures $4 \mathrm{C}-\mathrm{E}$ ). This enhanced tumor burden is not driven by increased MET or Wnt signaling (Supplemental Figure 8 and Figure 5). Even though IQGAP1 overexpression can enhance $\beta$-catenin activity in vitro (Figures 5B-C) and significantly increase cytosolic $\beta$-catenin protein in vivo (Figure 5D), we find that $\beta$-catenin and IQGAP1 co-localize at the cell membrane in $\mathrm{B}+\mathrm{M}+\mathrm{I}$ livers. This implies that higher IQGAP1 prevents $\beta$-catenin nuclear translocation (Figure $5 \mathrm{G}$ ) and is in line with a well-known mechanism regulated by IQGAP1 that enhances E-Cadherin- $\beta$-catenin complexes at the adherens junctions (Figures $5 \mathrm{~F}-\mathrm{G})[44,45,65,66]$.

HCC tumors are heterogenous and are not driven by a singular oncogenic pathway, and IQGAP1 is known to enhance multiple oncogenic pathways [28, 29, 67]. We found one such pathway in our model to be the Hippo/YAP signaling pathway. IQGAP1 is known to interact with and modulate YAP activity $[48,49]$. We found significantly elevated expression of Birc5, a target strongly linked to YAP activity [47], when IQGAP1 is overexpressed in the B+M model (Figure $5 E)$. In addition, we also found more $Y A P$ protein in the nucleus of $B+M+I$ samples compared to $B+M($ Figure $6 A$ ) and its targets remained unchanged when comparing $B+M$ to $B+M+I$ samples (Figure 6B). This data matched well with recent findings that most YAP downstream proteins are unaltered in HCC except for NUAK2 (Figure 6C). To evaluate the translational relevance of this findings, we mined TCGA database. We found 7 patients with IQGAP1 ${ }^{\text {High }} / \mathrm{NUAK} 2^{\text {High }}$ expression (Figure 6D), who not only have increased proliferative signaling pathways but also exhibit poor survival (Supplemental Figure 8 and Figure 6E). Additionally, these patient samples have no activating CTNNB1 mutations (data not shown), which indicates therapies targeting IQGAP1 and/or NUAK2 may be more beneficial. Globally, there were about 953,000 liver 
cancer cases in 2017 [68]. Considering that $1.9 \%$ of patients in the TCGA cohort have increased IQGAP1 and NUAK2 expression, this equates to roughly 18,000 patients per year worldwide. This might be an underestimate due to our use of TCGA mRNA expression data, since its known that $60-80 \%$ of HCCs display elevated IQGAP1 protein expression [11-14].

In summary, we show that overexpression and deletion of IQGAP1 promote hepatic tumorigenesis through two distinct mechanisms- by YAP signaling or by increased MET expression respectively. Such paradoxical findings have been reported in other key proteins involved in tumorigenesis, including NF-KB, JNK kinases, STAT3, MET, and $\beta$-catenin [69]. Our results indicate that IQGAP1 may function as a rheostat to balance pro-proliferative signals and that too little and too much may tip the balance towards uncontrolled proliferation. Therefore, targeting domain-specific interactions of IQGAP1 may be useful strategy to combat hepatic tumorigenesis.

\section{Methods}

Animals. The Institutional Care and Use Committee of approved all mouse experiments. $\operatorname{lqgap} 1^{+/+}, \operatorname{lqgap} 1^{+/-}$, and $\operatorname{lqgap} 1^{-/-}$mice maintained on a 129/SVJ background (129Iqgap $\left.1^{\text {tm1aber }} \mathrm{NSJ}\right)$ were used for all diethylnitrosamine (DEN) experiments. These mice were generated in Dr. Andrew Bernards's laboratory (Massachusetts General Hospital, Boston, Massachusetts, USA) and were obtained from Dr. Valentina Schmidt (Stony Brook University, New York, USA). FVB/NJ mice were obtained from Jackson Labs (Bar Harbor, ME). Animals were either housed at the University of Illinois at Urbana-Champaign on conventional racks or the University of Pittsburgh in Optimice cages (AnimalCare Systems, Centennial, CO) with SaniChip Coarse bedding (P. J. Murphy, Montville, NJ) at $24^{\circ} \mathrm{C}$ on a $12 / 12$ hour light/dark cycle with lights on starting at 5AM CST, corresponding to zeitgeber time (ZT) 0. Genotype was confirmed by PCR analysis of genomic DNA from tail clips. Mice were allowed ad libitum access to water and either Teklad F6 Rodent Diet (Envigo 8664) or standard mouse chow (LabDiet, St. Louis, 
MO, Purina ISO Pro Rodent 3000). Mice were provided huts and running wheels for enrichment. All animals were sacrificed between 9 am and noon daily.

Mice experiments. Male $(\mathrm{n}=77)$ and female $(\mathrm{n}=15)$ littermate $\operatorname{lqgap} 1^{+/+}$, lqgap $1^{+/}$, and Iqgap $1^{-/-}$mice were injected with $5 \mathrm{mg} / \mathrm{kg}$ DEN (N0258, Sigma-Aldrich) in sterile $1 \mathrm{x}$ PBS at 12-15 days of age via intraperitoneal injection (10 $\mu \mathrm{L} / \mathrm{g}$ body weight). Mice were sacrificed at both 20 weeks and 50 weeks after administration to assess tumor burden.

Six to eight-week-old male FVB/NJ mice were used for hydrodynamic tail vein injections. Mice were injected with 20 mg of pT3-EF5 $\alpha$-hMet-V5, pT3-EF5 $\alpha$-S45Y- $\beta$-catenin-Myc, or pT3-EF5aIQGAP1-HA, or combination of EF5 $\alpha-h M e t-V 5$ and pT3-EF5 $\alpha-S 45 Y-\beta$-catenin-Myc, or combination of EF5 $\alpha$-hMet-V5, pT3-EF5 $\alpha-S 45 Y-\beta-c a t e n i n-M y c$ and pT3-EF5 $\alpha-I Q G A P 1-H A$ along with the sleeping beauty transposase $(\mathrm{SB})(0.8 \mathrm{mg})$ in a ratio of $25: 1$. Injections were diluted into a total of $2 \mathrm{~mL}$ of normal saline $(0.9 \% \mathrm{NaCl})$ and injected into the lateral tail vein in 5 to 7 seconds. See Supplemental Table 2 for construct information.

At the time of sacrifice, blood was collected by retro-orbital bleeding, and serum was separated by centrifugation and immediately stored at $-80^{\circ} \mathrm{C}$ in opaque tubes. Liver, gonadal white adipose tissue, spleen, and quadriceps tissues were collected, weighed, and flash frozen for analysis. A piece of each liver/tumor and the lungs were fixed in $10 \%$ formalin for histological analysis.

Body weight and LW/BW. Livers from experimental animals were excised, washed in PBS and weighed. The percentage of the weight occupied by the liver was determined by dividing the liver weight by the total body weight of the mouse. 
Cell Lines. Human HepG2, Hep3B, Snu-449 and Huh7 hepatoma cell lines were obtained from American Type Cell Culture (ATCC). HepG2, Hep3B and Huh7 were maintained in 10\% fetal bovine serum (FBS) (Atlanta Biologicals) in DMEM. Snu-449 cells were maintained in 10\% FBS in RPMI-1640. Cells were incubated at $37^{\circ} \mathrm{C}$ in a humidified $5 \%$ carbon dioxide atmosphere.

Constructs Used. pEGFP-IQGAP1 was a gift from David Sacks (Addgene plasmid \# 30112). Using this construct, an HA tag was added to IQGAP1 and cloned via Gateway PCR (Invitrogen, Carlsbad, CA) into a pT3-EF5a vector. Additional constructs used can be found in Supplemental Table 2.

Luciferase assay. Cell lines were transfected simultaneously with $400 \mathrm{ng}$ TOPFlash firefly luciferase reporter and 100 ng Renilla luciferase constructs alongside either siRNA or expression constructs listed in Supplemental Table 2 using Lipofectamine 2000 (Life Technologies). Transfected cells were harvested after $72 \mathrm{~h}$ and processed with Dual-Luciferase Reporter Assay kit (Promega). Luciferase activity was detected with an Infinite M200 PRO microplate reader (Tecan, Männedorf, Switzerland). Relative luciferase activities of transfected plasmids are represented as the activity of firefly luciferase activity normalized to Renilla activity.

Lentivirus production and shRNA knock down. Lentiviruses packaged with shRNA were produced as previously described [70]. Constructs used are listed in Supplemental Table 2. HepG2 cells were infected with $25 \mu \mathrm{L}$ of lentivirus-containing medium expressing either shScrambled or shIQGAP1 constructs and infected cells were selected by culturing cells in $1 \mu \mathrm{g} / \mathrm{mL}$ puromycin for 96 hours. Cell lysates were processed for RNA expression analysis or western blotting using TriZOL or RIPA buffer, respectively. 
Histology. Liver samples were fixed in formalin for $>24$ hours. They were then processed and embedded in paraffin wax. Four or five $\mu \mathrm{m}$ sections were cut. For immunohistochemistry, sections were deparaffinized using xylene and graded ethanol (100-95\%) washes and incubated in citric-acid based antigen retrieval (Vector, Burlingame, CA). Following antigen retrieval, liver sections were treated with $3 \%$ hydrogen peroxide to quench endogenous peroxidase and blocked with either $5 \%$ normal goat serum in $5 \%$ bovine serum albumin in TBST or Avidin/Biotin blocking solution (Vector SP-2001). Slides were incubated with primary antibody followed by biotinylated secondary antibody or HRP-conjugated secondary antibody (concentrations indicated in Supplemental Table 3). Either avidin-conjugated peroxidase (Vector ABC kit PK-6100) with ImmPACT DAB Peroxidase Substrate (Vector SK-4105) or DAB Peroxidase (HRP) Substrate Kit (SK-4100, Vector Labs) were used to visualize stained tissues. Sections were counterstained with Modified Harris Hematoxylin (Richard Allen 72711) dehydrated with ethanol and xylene washes and mounted with Permount (Fisher).

Briefly, H\&E staining was performed after deparaffinization. Slides were first stained with hematoxylin and rinsed with water followed by dipping in $5 \%$ glacial acetic acid. Shandon's Bluing Reagent (ThermoScientific, Kalamazoo, MI) was used to retain hematoxylin counterstain. Slides are then dipped in Eosin for 1 minute and dehydrated with ethanol and xylene washes followed by mounting with Permount.

\section{RNA isolation, quantitative reverse transcriptase polymerase chain reaction (qRT-PCR)}

and PCR. Total RNA from fresh liver and tumor samples collected at sacrifice was extracted using TRIzol solution (Invitrogen) and subjected to qRT-PCR to quantify the expression of protein-coding genes. A260/280 and bleach RNA gel were used to assess RNA quality. RNA with an A260/280 > 2.0 and a 28S/18S RNA ratio of approximately 2 was used for further analysis. Complementary DNA (cDNA) synthesis and qRT-PCR were performed either as 
previously described [24] or using M-MLV (Life Technologies) followed by q-PCR performed with SYBR Green PCR Master Mix (Life Technologies), Bullseye EvaGreen PCR Master Mix (Midwest Scientific), or Taqman probes (Life Technologies) with Taqman Universal Master Mix II (Life Technologies). Primer sequences and Taqman probe IDs are described in Supplemental Table 4. Reactions were performed using a StepOnePlus System (Life Technologies). Relative expression was calculated using the $\Delta \Delta \mathrm{CT}$ method. Gapdh was used as housekeeping gene. Taqman probe IDs and primer sequences are listed below. Reactions were performed using a StepOnePlus System (Life Technologies).

DNA mutation analysis. Genomic DNA was isolated from DEN-induced liver tumors at 50 weeks post-treatment using QIAamp Fast DNA tissue kit (Qiagen). Fludigm technology was used to sequence the DNA using four primer sets (Supplemental table 4). The read files were demultiplexed by primer and then by sample. Fastp version 0.19 .5 was used to perform quality filtering and trimming of the raw reads. The files were qc-trimmed and aligned to the mouse reference genome version GRCm38.p6 with $\mathrm{NCBI}$ WebBlast in order to obtain the absolute coordinates in the forward strand version of the genome with bwa version 0.7.17 using default parameters. Bam-read count version 0.8 was run to generate the read counts at each of the locations identified. Then a customized R script was run to generate a summary of nucleotide frequencies at the codons of interest. The base pair with the lowest percent of reads matching the WT sequence was used to determine the prevalence of mutation at that codon in each sample. The raw sequence data have been uploaded to NCBI Bioproject (http://www.ncbi.nlm.nih.gov/bioproject/629000) and will be made public after publication.

Western blotting. Whole tissue protein lysates were prepared from approximately $50 \mathrm{mg}$ frozen tissue using sodium dodecyl sulfate (SDS)-based lysis buffer (50 mM Tris-HCl, pH 8.0, 10 mM 
EDTA, 1\% SDS) containing protease/phosphatase inhibitors (RIPA buffer). Lysates were removed to a fresh $1.5 \mathrm{ml}$ tube and centrifuged at $18.4 \mathrm{~g}$ for $10 \mathrm{~min}$. at $4^{\circ} \mathrm{C}$ in order to remove clear supernatant to a new $1.5 \mathrm{ml}$ tube while disposing of the pellet. Samples were stored at $80^{\circ} \mathrm{C}$ until utilization or determination of protein concentration via BCA protein assay (Fisher) to ensure equal protein concentrations for subsequent assays. For Western blot, $50-200 \mu \mathrm{g}$ total protein was loaded onto $8 \%-12 \%$ SDS-PAGE gels. Protein were transferred to Immobilon-P PVDF membrane (IPVH00010, Millipore) either for 1 hour at $100 \mathrm{~V}$ at $4^{\circ} \mathrm{C}$ or overnight at $35 \mathrm{~V}$ and $4^{\circ} \mathrm{C}$. After transfer, the membranes were blocked in either $5 \%$ non-fat dry milk or $5 \%$ BSA dissolved in Blotto $(0.15 \mathrm{M} \mathrm{NaCl}, 0.02 \mathrm{M}$ Tris $\mathrm{pH} 7.5,0.1 \%$ Tween in $\mathrm{dH} 2 \mathrm{O})$ followed by incubation with antibodies described in Supplemental Table 3. Membranes were exposed to SuperSignal West Pico Chemiluminescent Substrate (Thermo Scientific Pierce, Pittsburgh, PA) for 1-2 min. at room temperature and bands reflective of target proteins were viewed by ChemiDoc imaging system (Bio-Rad). Bands were quantified with ImageJ (National Institutes of Health, Bethesda, MD).

Nuclear/Cytoplasmic Fractioning. Whole liver samples were processed either by using the NE-PER Nuclear and Cytoplasmic Extraction kit (Life Technologies) following manufacturers recommendations or lysed using SDS-free subcellular fractionation buffer (20 mM HEPES, 10 $\mathrm{mM} \mathrm{KCl}, 2 \mathrm{mM} \mathrm{MgCl}$, $1 \mathrm{mM}$ EDTA, $1 \mathrm{mM} \mathrm{EGTA)} \mathrm{containing} \mathrm{protease/phosphatase} \mathrm{inhibitors.}$ Samples were manually agitated and incubated on ice for 30 minutes prior to centrifugation at 3000 RPM for 5 minutes at $4^{\circ} \mathrm{C}$. The supernatant containing the cytosolic fraction was moved to a fresh, $1.5 \mathrm{ml}$ tube while the pellet was washed $5 \mathrm{x}$ with subcellular fractionation buffer. After final wash, nuclear pellet was lysed using TBS with $0.1 \%$ SDS. Samples were stored at $-80^{\circ} \mathrm{C}$ until use in protein quantification and western blotting as previously described. 
Immunoprecipitation. Whole liver samples were lysed using immunoprecipitation lysis buffer (20 mM Tris-base, 150 mM NaCl, 1 mM EDTA, 1 mM EGTA, 1\% Triton-X pH 7.5) containing protease/phosphatase inhibitors. Samples were manually agitated and incubated on ice for 30 minutes prior to centrifugation at 15,000 RPM for 15 minutes at $4^{\circ} \mathrm{C}$. Supernatants were removed to a fresh $1.5 \mathrm{ml}$ tube while the pellet was discarded. Samples were stored at $-80^{\circ} \mathrm{C}$ until utilization or determination of protein concentration via BCA protein assay (Fisher) to ensure equal protein concentrations. Equal amounts of protein from respective groups were pooled together and precleared on ice using normal control mouse IgG (Life Technologies) for 30 minutes followed by incubating with A/G PLUS-Agarose beads (Santa Cruz) overnight at $4^{\circ} \mathrm{C}$ with gentle agitation. Samples were centrifuged for 5 minutes at 3000 RPM and supernatants were removed to a fresh $1.5 \mathrm{ml}$ tube together with $5 \mu \mathrm{g}$ of $\beta$-catenin monoclonal antibody (Supplemental Table 3) overnight at $4^{\circ} \mathrm{C}$ with gentle agitation. A/G PLUS-Agarose beads were applied to each sample overnight at $4^{\circ} \mathrm{C}$ with gentle agitation. The supernatant was then removed from beads via centrifugation to a fresh $1.5 \mathrm{ml}$ tube and the pellet was washed four times with PBS containing protease/phosphatase inhibitor to prevent disruption of delicate protein-protein interactions. Samples were then processed for western blotting as previously described.

Data Analysis. RNA-Sequencing gene expression data from the Hepatocellular Carcinoma TCGA Firehose Legacy dataset was downloaded from the cbioportal (cbioportal.org) [71, 72]. Patients were separated into two cohorts, those that have amplified IQGAP1 and NUAK2 mRNA expression fitting a cutoff $z$-score threshold \pm 2 , and those without. Gene expression data were analyzed through the use of IPA (QIAGEN Inc., https://www.qiagenbioinformatics.com/products/ingenuitypathway-analysis) using an experimental $p$-value $>0.02$ and a false discovery rate q-value $>0.04$ [73]. Canonical pathway amplification/downregulation was determined using a -log(p-value $)<2.3$ and a z-score 
$<2.5$ while thresholding at 0.05 . Fisher's exact test was used to determine significance of pathway alterations. Finally, pathways were filtered for relevance to liver biology and disease pathogenesis. For survival analysis, clinical data from the TCGA were analyzed to determine overall survival calculated from diagnosis date to the death date or date of last contact taking censoring into consideration. Overall survival was then calculated for the patients using KaplanMeier methods.

Statistical Analysis. Data are expressed as mean \pm SD or SEM. All statistical analyses were performed using GraphPad Prism software version 7. For contingency data, $\mathrm{x}^{2}$ test was used to compare 3 groups and Fisher's exact test was performed to assess differences between 2 groups. One-way ANOVA with Bonferroni post-test was performed to compare 3 groups while two-way ANOVA with Tukey's post-test was used to assess differences between two paired tissues (liver and tumor) in three groups. Asterisks indicate a statistically significant difference between groups. Significance is defined as $P<0.05$. Outliers were determined by Grubbs' test and were removed from analysis along with any paired data.

Study Approval. Animal studies were approved by the Institutional Animal Care and Use Committees at the University of Illinois at Urbana-Champaign and University of Pittsburgh. All animal studies were carried out as outlined in the Guide for the Care and Use of Laboratory Animals [74].

Acknowledgements. IPA licensed through the Molecular Biology Information Service of the Health Sciences Library System, University of Pittsburgh was used for data analysis.

\section{Authors' Contributions}


Writing, review, and/or revision of the manuscript: H.L.E., E.R.D., A.W.D., S.A .

Technical, or material support (experimental design, execution, data and statistical analysis):

H.L.E., E.R.D., J.T, S.P.M., A.W.D, S.A .

Study supervision: A.W.D., S.A.

\section{Financial support}

S.A.: R01 DK113080, ACS132640-RSG

A.W.D.: R01 DK103645

H.L.E.: F30 CA206495 
Figures and Figure Legends

A

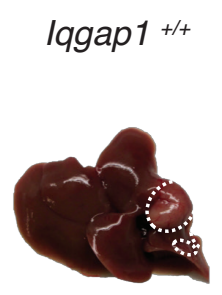

lqgap $1^{-/}$

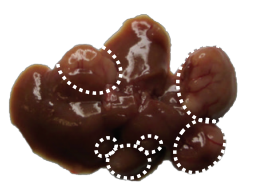

- $\operatorname{lqgap} 1^{-/-}$

○ $\operatorname{lqgap} 1^{+/+}$
B

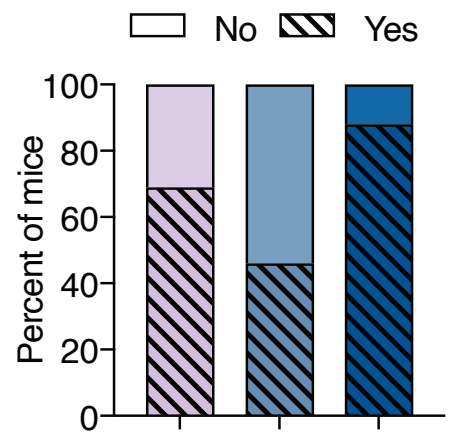

$\chi^{2}: \quad P=0.0242$
C Tumor Multiplicity

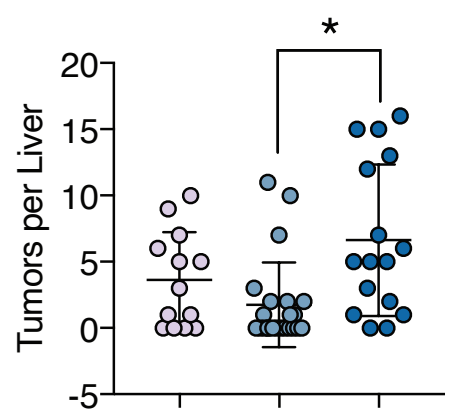

ANOVA: $\quad P=0.0285$

F
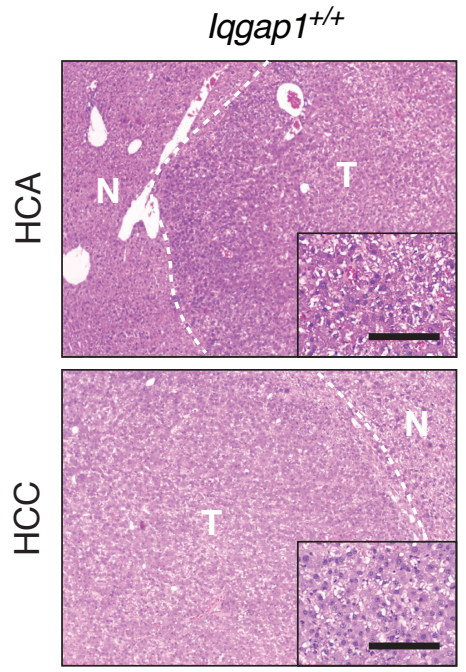

D Largest Tumor Size

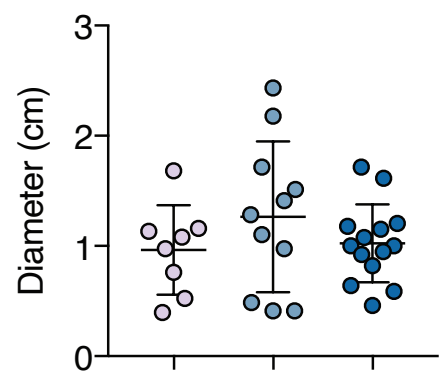

ANOVA: $\quad P=0.3578$
E Liver to Body Weight

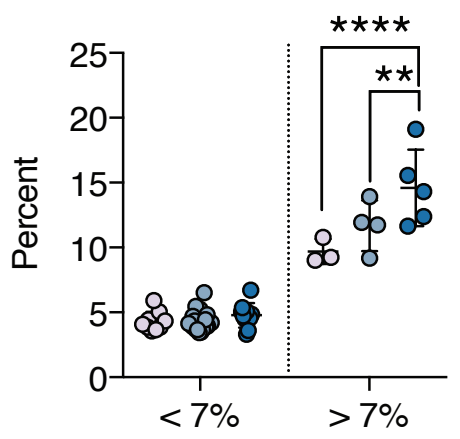

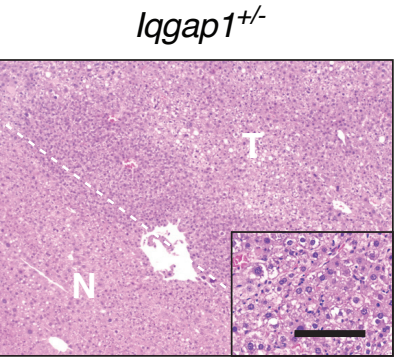
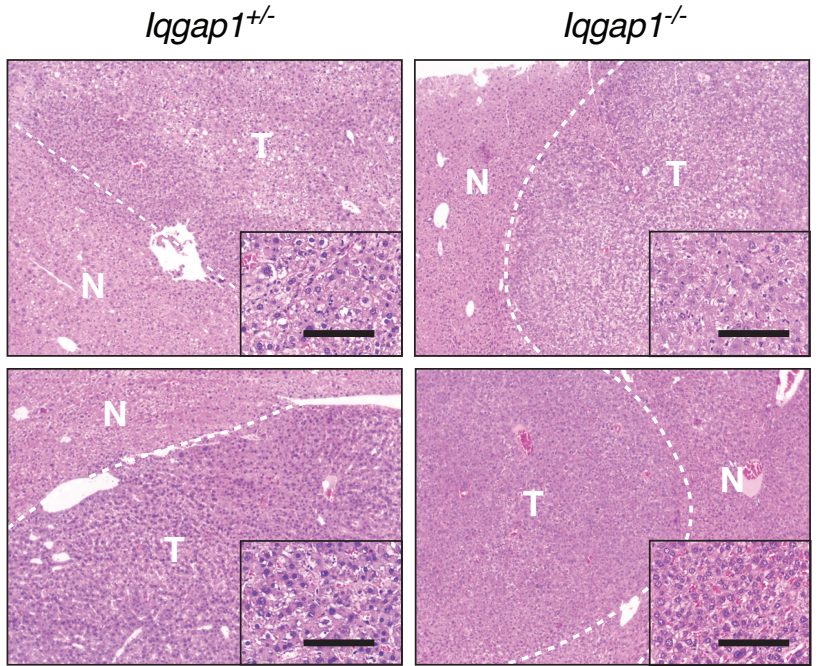
Figure 1. IQGAP1 deletion promotes liver tumor development. Male $\operatorname{lqgap} 1^{+/+}(\mathrm{n}=13)$,

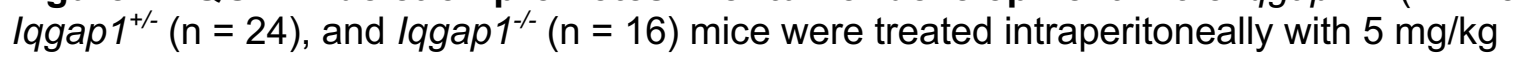
diethylnitrosamine (DEN) in sterile PBS at 12-15 days of age. At 1 year, tumor burden was assessed. (A) Representative photos of gross liver. Tumor nodules are indicated by a white dashed border. Scale bar is $1 \mathrm{~cm}$. (B) Tumor incidence based on presence of visible liver nodules. (C) Tumor multiplicity was measured by counting the number of visible tumors per liver. (D) Tumor size was assessed by measuring the diameter of the largest visible tumor. (E) Liver weight normalized to body weight divided into low and high groups. (F) Representative H\&E images of normal liver tissue, hepatocellular adenoma (HCA), and hepatocellular carcinoma $(\mathrm{HCC})$ in tumor-bearing $\operatorname{lqgap} 1^{+/+}$, Iqgap $1^{+/}$, and $\operatorname{lqgap} 1^{-/-}$mice $(\mathrm{n}=9,8$, and 12 mice per group, respectively). Scale bar is $200 \mu \mathrm{m} ; 100 \mu \mathrm{m}$ (inset). Values are displayed as mean \pm $\mathrm{SD}$. For tumor incidence, $\mathrm{X}^{2}$ test was used to determine significance between all 3 groups. For tumor multiplicity and largest tumor size, one-way ANOVA with Bonferroni's multiple comparison test was used to determine significance between groups. For liver weight and liver to body weight ratio, two-way ANOVA with Bonferroni's multiple comparison test was used to determine significance between groups. Significance is indicated by ${ }^{*} P<0.05,{ }^{* *} P<0.01$, ${ }^{* * *} P<0.001$, ${ }^{* * * *} P<0.0001$. 
A

A Non-Tumor

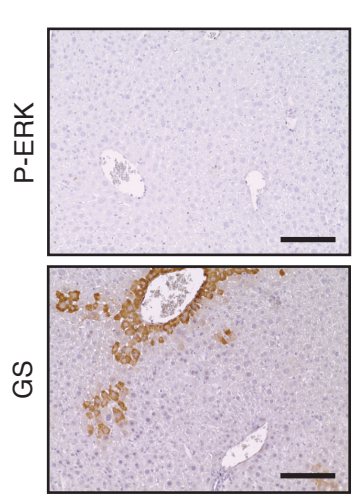

B

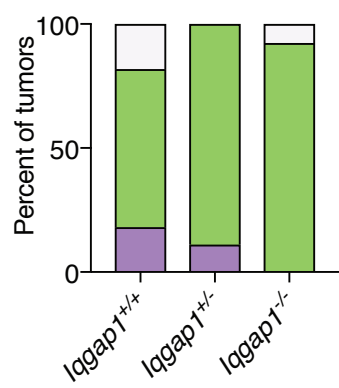

D
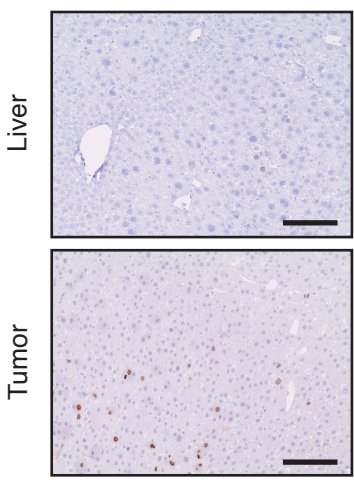

GS and P-ERK Negative

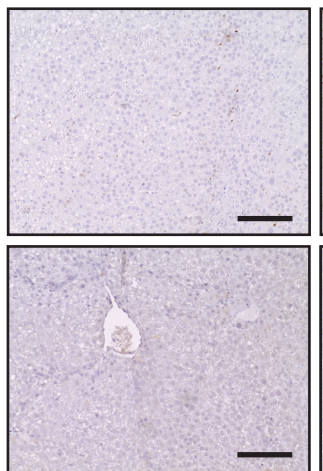

C

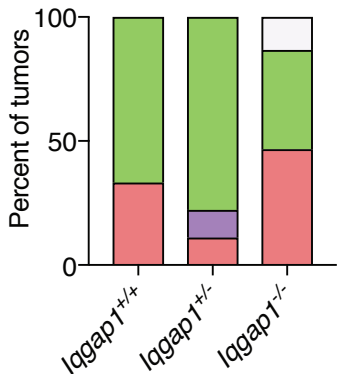

$\operatorname{lqgap} 1^{-1}$

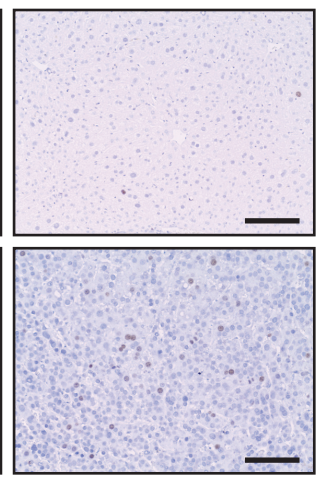

Tumor
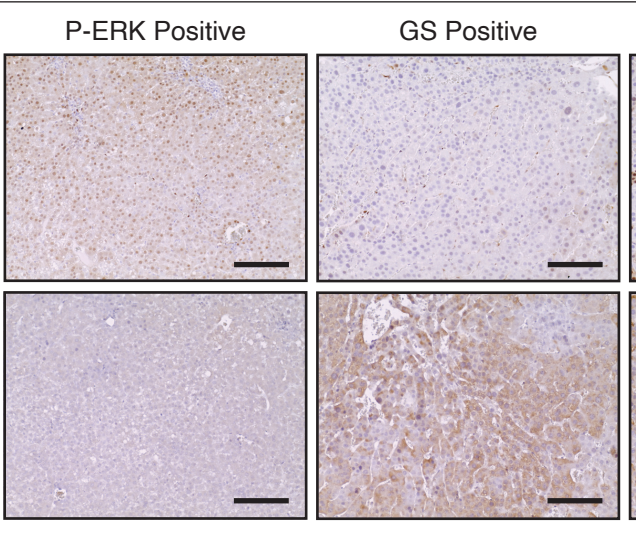

GS and P-ERK Positive

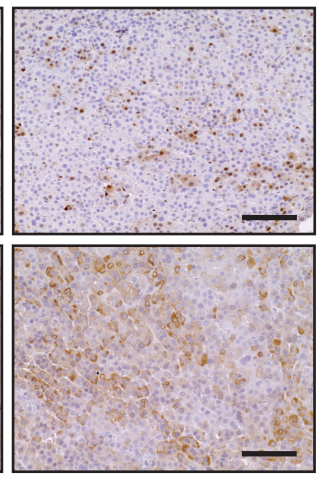

$\square$ GS and P-ERK Negative

$\square$ P-ERK Positive

$\square$ GS Positive

$\square$ GS and P-ERK Positive

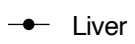

-o- Tumor

Figure 2. DEN-induced tumors are either P-ERK or GS positive. (A) Representative immunohistochemistry images of anti-P-ERK and anti-GS staining in normal liver tissue and tumor tissue classified as either GS and P-ERK negative, P-ERK positive, GS positive, or GS and P-ERK positive. Scale bar is $100 \mu \mathrm{m}$. (B) The percent of HCA tumors with each molecular classification ( $\mathrm{n}=12,9$, and 15 lqgap $1^{+/+}$, Iqgap $1^{+/-}$, and lqgap $1^{-/}$tumors, respectively). (C) The percent of HCC tumors with each molecular classification $\left(\mathrm{n}=11,9\right.$, and 13 lqgap $1^{1 /+}$, Iqgap ${ }^{1 / \text {, }}$, and Iqgap $1^{-/}$tumors, respectively). (D) Representative images of anti-Ki-67 immunohistochemistry staining in liver and tumor tissue of Iqgap $1^{+/+}$, Iqgap $1^{+/}$, and lqgap $1^{-/}$ animals ( $n=9,8$, and 12 mice per group, respectively). Scale bar is $100 \mu \mathrm{m}$. (E) Quantification of proliferating (Ki-67 positive) nuclei in liver and tumor tissue. Values are displayed as mean \pm SD. Two-way paired ANOVA with Tukey's multiple comparisons test was used to assess 
bioRxiv preprint doi: https://doi.org/10.1101/2020.05.29.124404; this version posted May 31, 2020. The copyright holder for this preprint (which

was not certified by peer review) is the author/funder, who has granted bioRxiv a license to display the preprint in perpetuity. It is made available under aCC-BY-NC-ND 4.0 International license.

differences between groups. Significance is indicated with * $P<0.05$, ${ }^{* *} P<0.01$, ${ }^{* *} P<0.001$, ${ }^{\star * * *} P<0.0001$. 

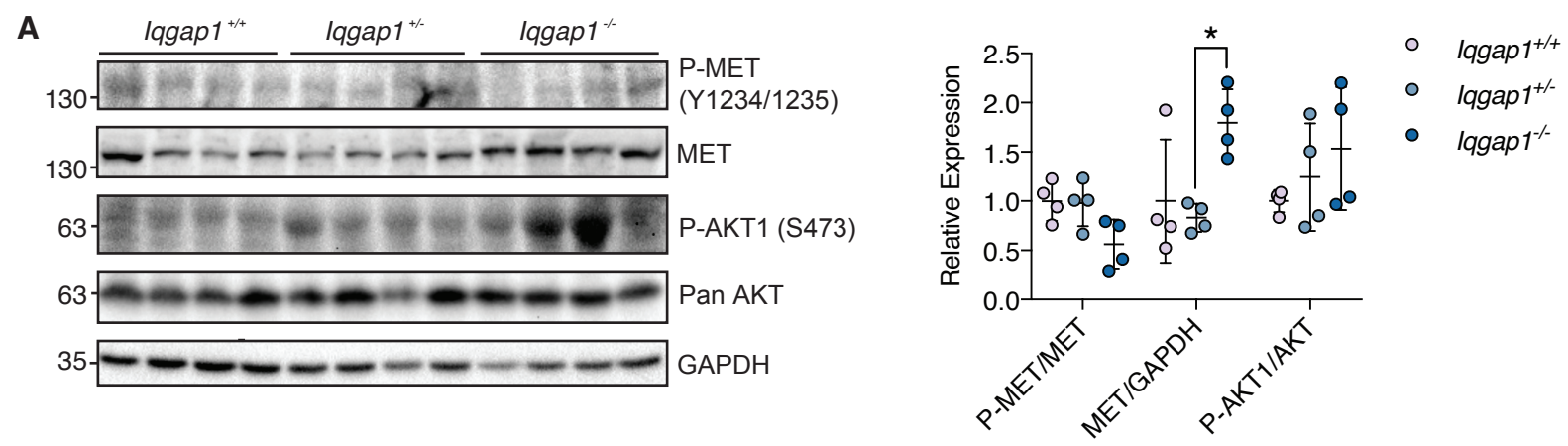

B
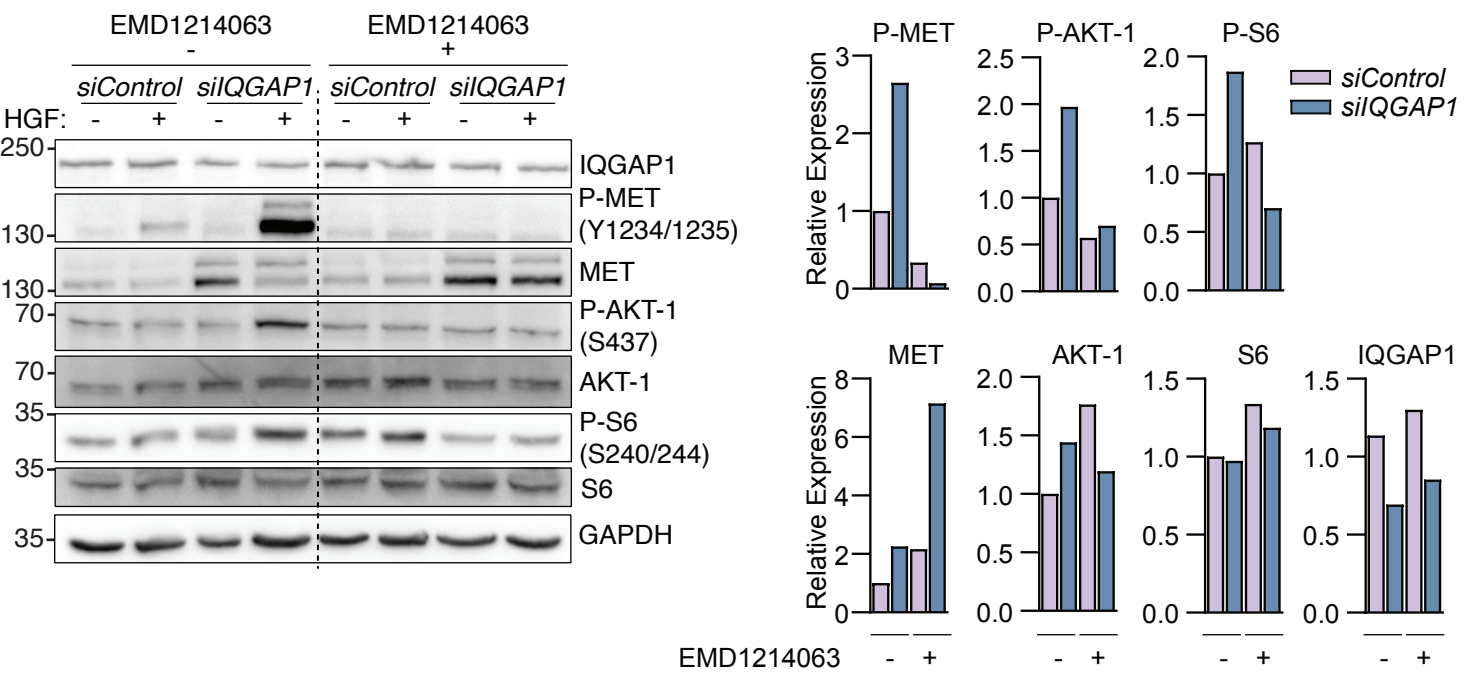

C

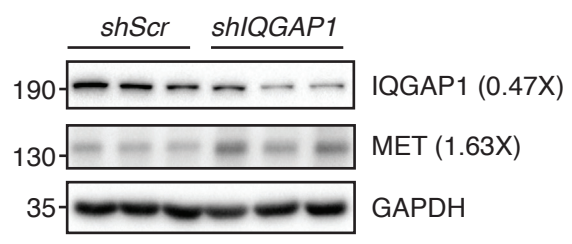

○ $s h S c r$

- ShIQGAP1
D

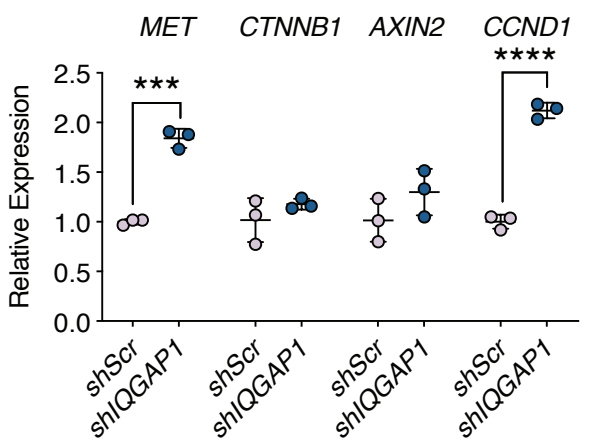

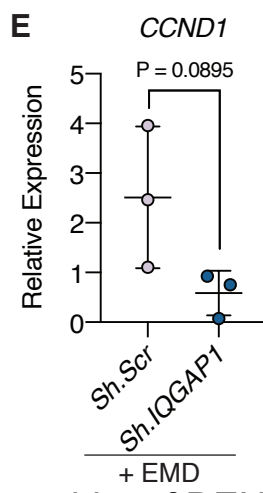

Figure 3. IQGAP1 loss enhances MET expression and activation. (A) Immunoblot of DENtreated lqgap $1^{+/+}$, Iqgap $1^{+/}$, and lqgap $1^{-/-}$tumor extracts. Each lane contains extracts from a single mouse. Quantification of immunoblots by densitometry provided at right. (B) Snu-449 HCC cells were transfected with either Control or IQGAP1 siRNA for 48 hours. Cells were then serum starved overnight and treated with \pm HGF $(50 \mathrm{ng} / \mu \mathrm{L})$ and \pm EMD1214063 (10 nM) for 4 hours prior to harvest. Whole cell lysates were immunoblotted for phosphorylated and total forms of specific targets and normalized to GAPDH. Conditions are representative of 3 independent experiments pooled together. Quantification of immunoblots with respect to only HGF+ condition by densitometry shown at right. (C) Immunoblot of HepG2 cells infected with lentivirus expressing shScrambled (shScr) and shIQGAP1. (D-E) Gene expression of HepG2 cells infected with shScr and shIQGAP1 (D) and treated with EMD1214063 (E) normalized to Gapdh expression. Values are displayed as mean \pm SD. To compare 2 groups, Student's t-test 
bioRxiv preprint doi: https://doi.org/10.1101/2020.05.29.124404; this version posted May 31, 2020. The copyright holder for this preprint (which

was not certified by peer review) is the author/funder, who has granted bioRxiv a license to display the preprint in perpetuity. It is made available under aCC-BY-NC-ND 4.0 International license.

was performed. Two-way paired ANOVA with Tukey's multiple comparisons test was used to assess differences between groups. Significance is indicated with ${ }^{*} P<0.05,{ }^{* *} P<0.01,{ }^{* * *} P$ $<0.001,{ }^{* * *} P<0.0001$. 
bioRxiv preprint doi: https://doi.org/10.1101/2020.05.29.124404; this version posted May 31, 2020. The copyright holder for this preprint (which was not certified by peer review) is the author/funder, who has granted bioRxiv a license to display the preprint in perpetuity. It is made available under aCC-BY-NC-ND 4.0 International license.

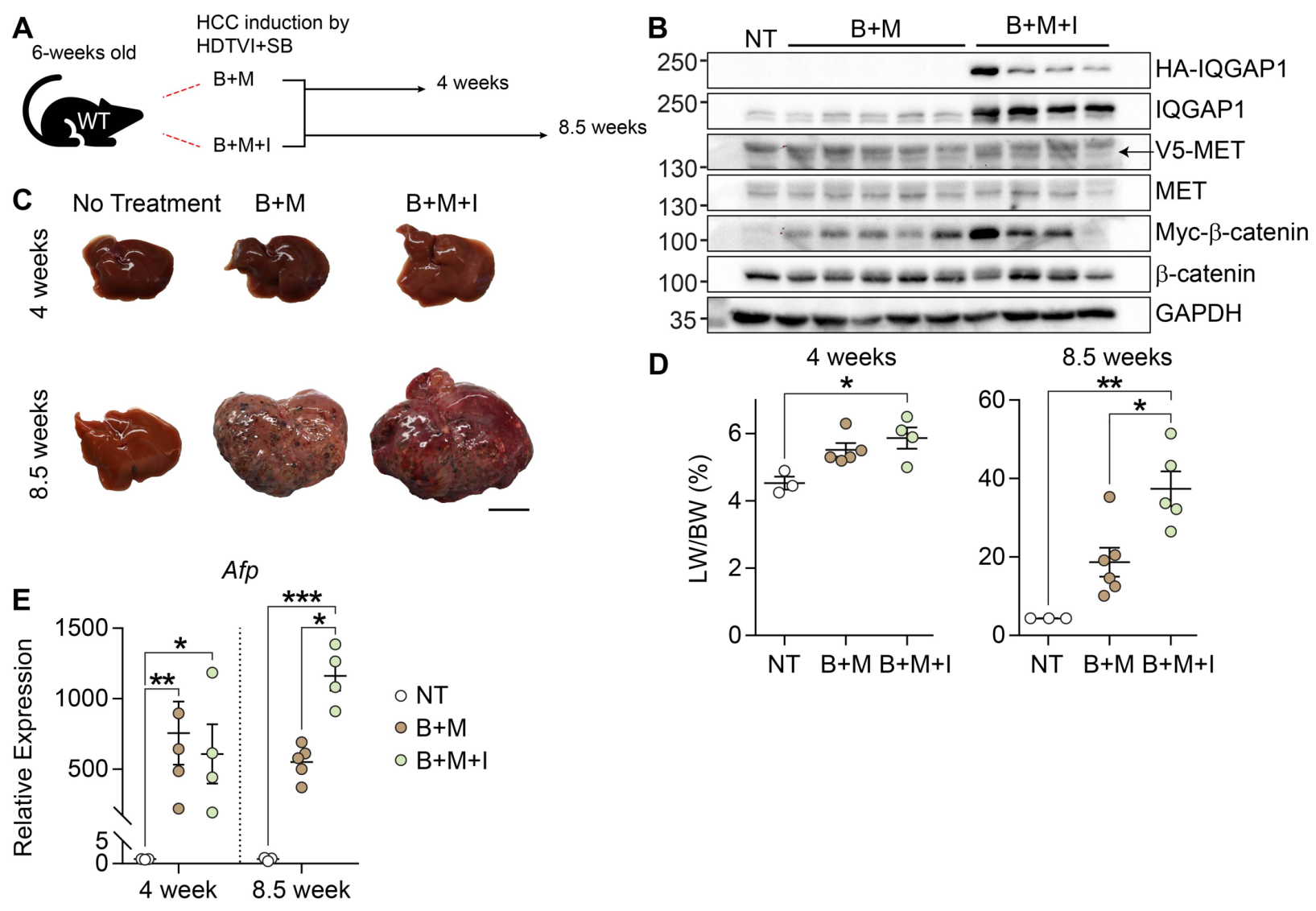

\section{F}

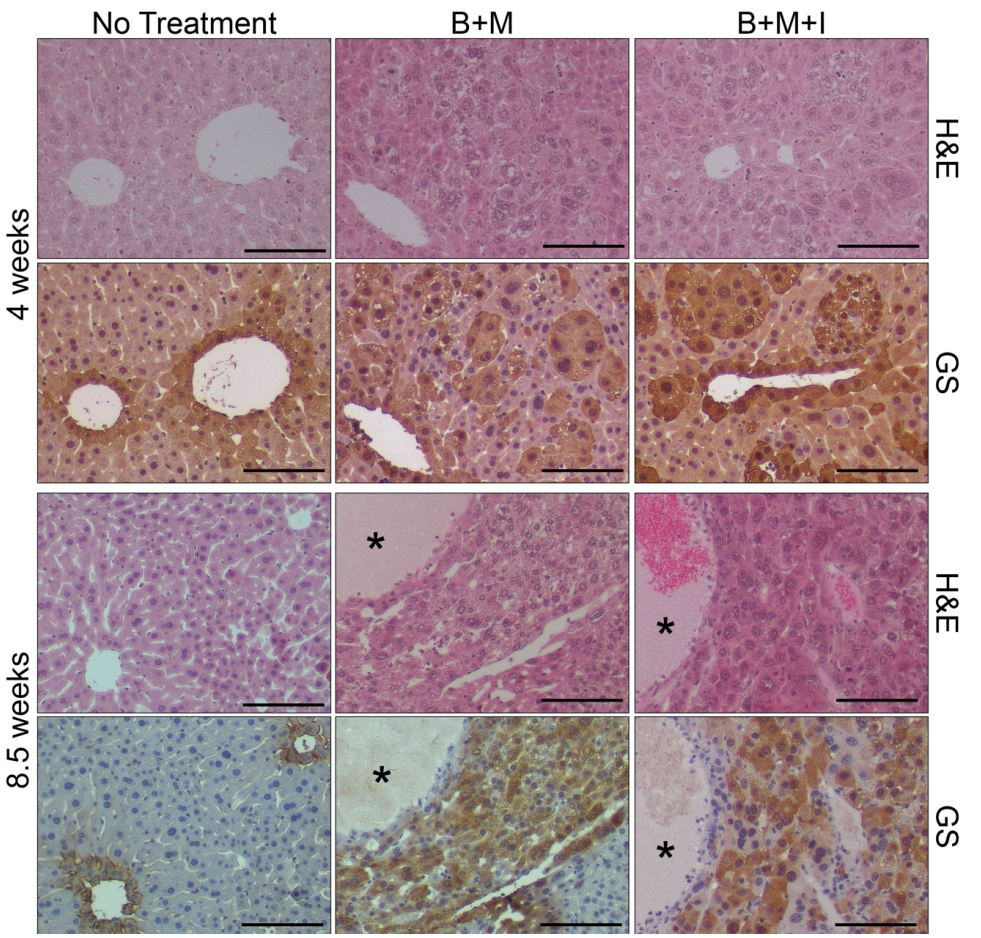


Figure 4. IQGAP1 overexpression increases HCC development in $B+M$ transposon system (A) Experimental design for Sleeping Beauty Transposase groups injected with either $\mathrm{B}+\mathrm{M}$ or $\mathrm{B}+\mathrm{M}+\mathrm{l}$ and harvested after 4 or 8.5 weeks. $(\mathrm{B})$ Whole liver protein lysates isolated after 4 weeks and analyzed by western blot to detect epitope tagged (HA, V5 or Myc) and total IQGAP1, MET, and $\beta$-catenin; normalized to GAPDH. (C) Representative livers from nontreated, $\mathrm{B}+\mathrm{M}$, and $\mathrm{B}+\mathrm{M}+\mathrm{I}$ groups. Macroscopic disease is visible as black or white lesions. (D) Liver weight to body weight (LW/BW) ratio. (E) Afp expression corrected for Gapdh. (F) Serial liver sections from 4-week and 8.5-week mice stained with H\&E and GS (brown) to identify $\beta$ catenin driven HCCs. Necrotic regions are marked by asterisks. Graphs show mean \pm SEM and dots represent individual mice. Two-way paired ANOVA with Tukey's multiple comparisons test was used to determine significance between groups. Significance is indicated with ${ }^{*} P<0.05,{ }^{* *}$ $P<0.01,{ }^{* *} P<0.001$. Gross morphology scale bar $(\mathrm{C})$ is $2.5 \mathrm{~cm}$ and histology scale $(\mathrm{F})$ bars are $100 \mu \mathrm{m}$. 
A

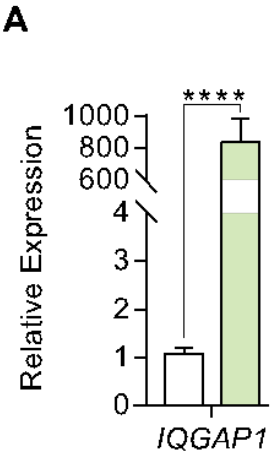

B

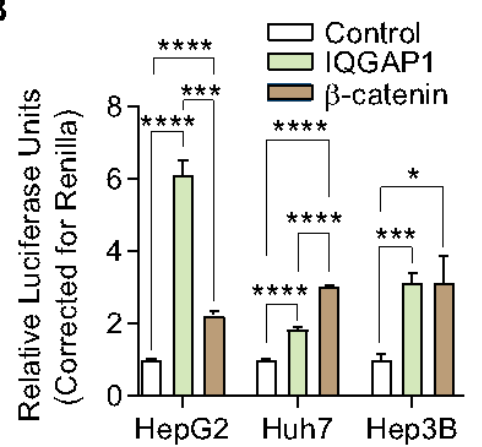

C

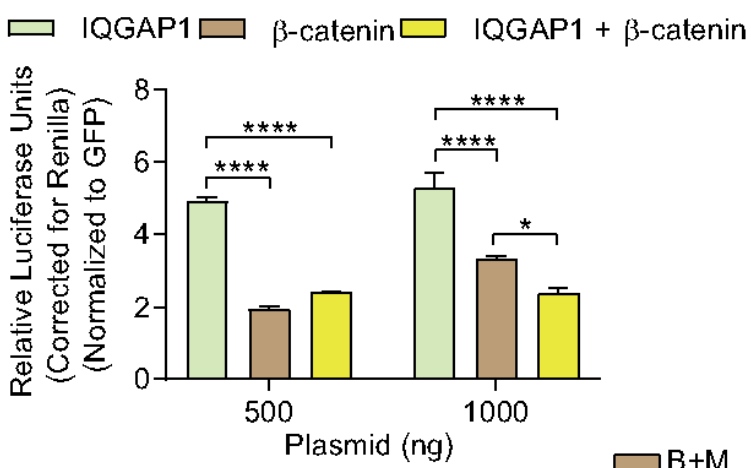

D
Cytosolic:

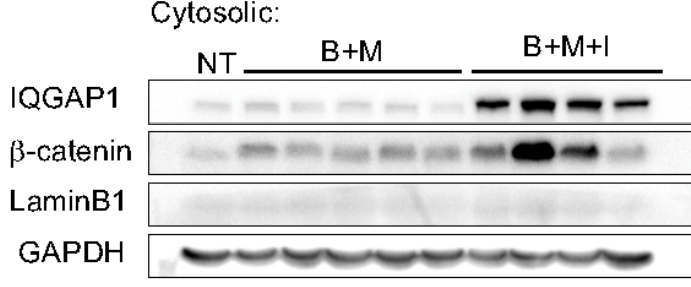

E

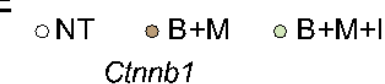

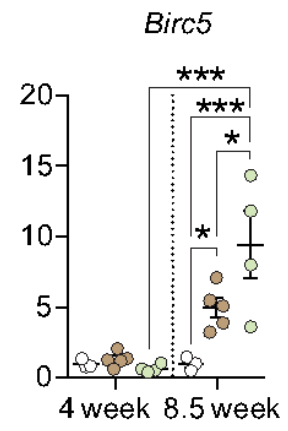

Nuclear

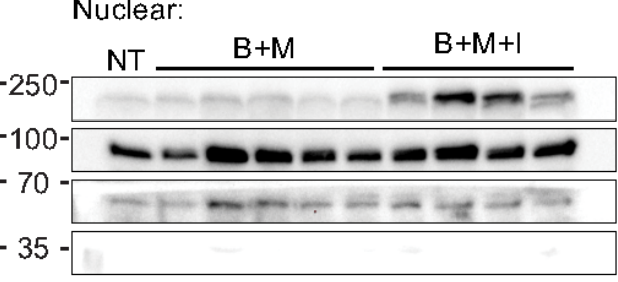

Lect2
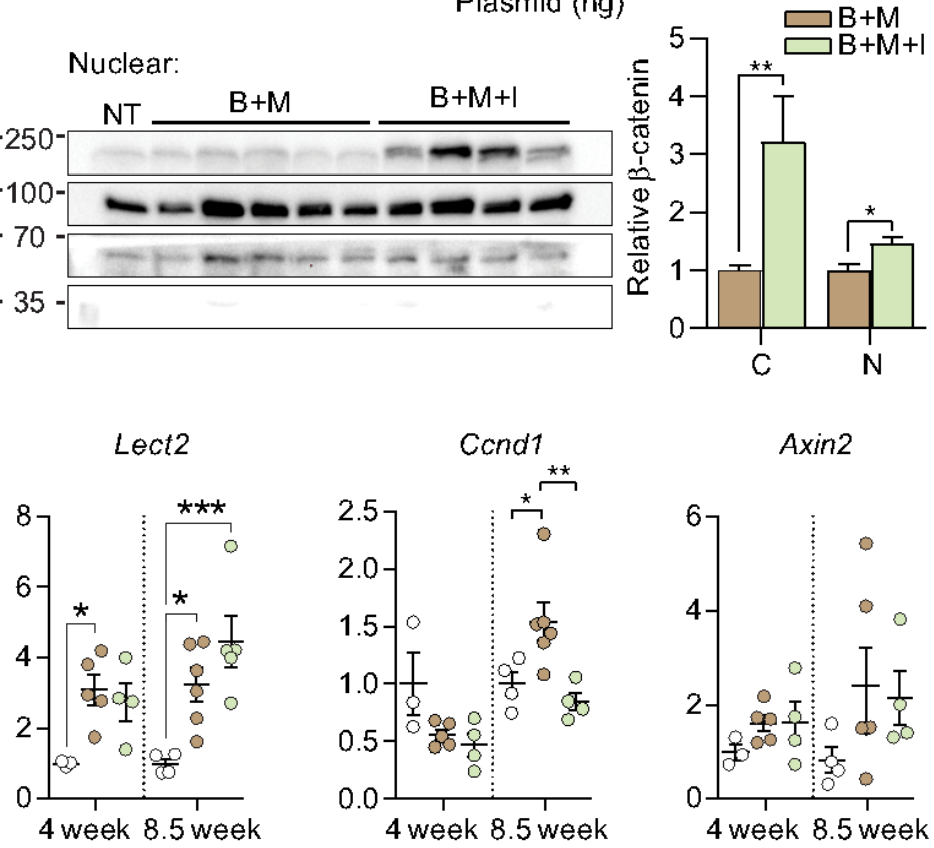

Axin2

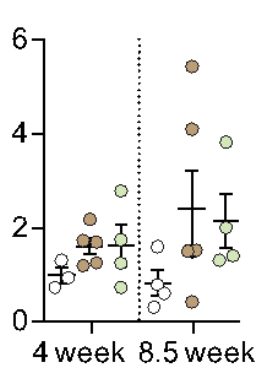

F

G

IP: $\beta$-catenin

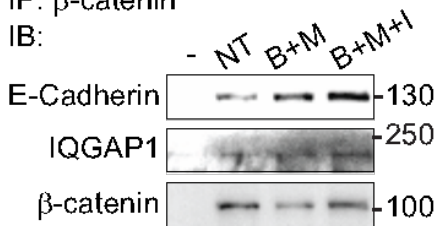

Hoechst

Met-V5

ß-catenin-Myc IQGAP1-HA

Merge

NT

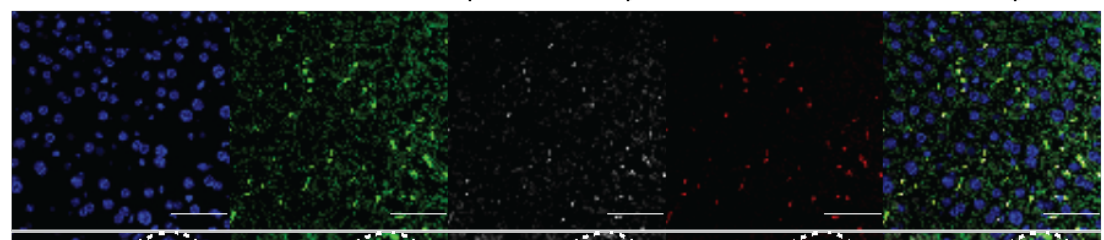

Input:

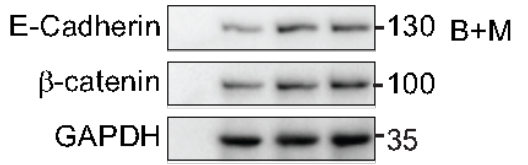

$\mathrm{B}+\mathrm{M}+\mathrm{I}$

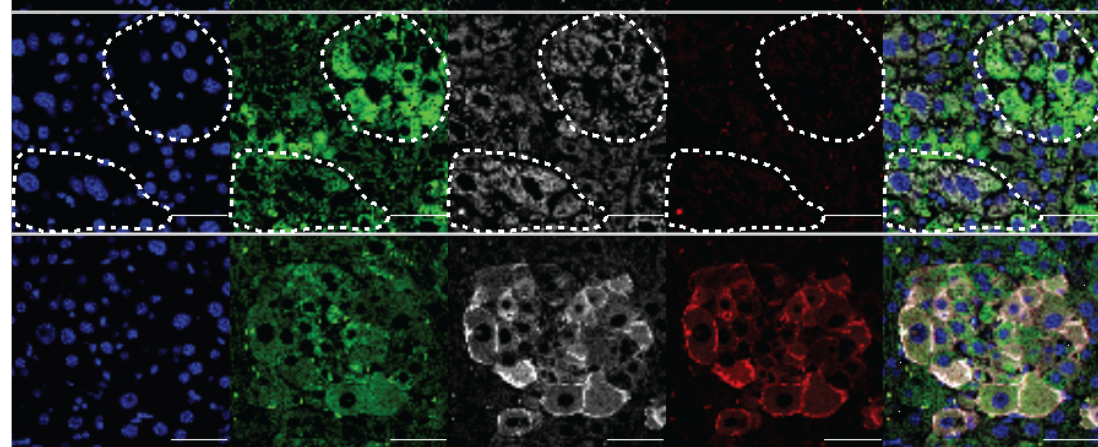

Figure 5. IQGAP1 overexpression does not induce Wnt/ $\beta$-catenin signaling in vivo. (A) HepG2 cells were transfected with GFP-control or IQGAP1 constructs for 72 hours. Expression for IQGAP1 was normalized to B2M. (B) HepG2, Huh7 and Hep3B cell lines were transfected 
with control, IQGAP1, or $\beta$-catenin expression constructs for 72 hours. Wnt/ $\beta$-catenin activity was measured by the TOPFlash luciferase reporter assay and corrected for Renilla luciferase. (C) HepG2 cells were transfected with GFP, IQGAP1, or $\beta$-catenin expression constructs for 72 hours. Additionally, cells were also transfected with IQGAP1 and $\beta$-catenin constructs together. $W n t / \beta$-catenin activity was measured and analyzed as previously described. (D) Cytosolic and nuclear protein from whole livers (4 week NT, $B+M$ and $B+M+I)$ were analyzed for IQGAP1 and $\beta$-catenin. Cytosolic protein was normalized to GAPDH and nuclear protein normalized to LaminB1. GAPDH and LaminB1 show purity of cytosolic or nuclear fractions, respectively. (E) Expression of Ctnnb1 and Wnt/B-catenin target genes Birc5, Lect2, Ccnd1 and Axin2 in whole livers from 4- and 8.5-week samples, normalized to Gapdh. (F) Whole liver lysates from NT $n=3$, $B+M n=5$, or $B+M+I n=4$ were pooled and immunoprecipitated (IP) for $\beta$-catenin then immunoblotted (IB) for E-Cadherin, IQGAP1 or $\beta$-catenin. Sample inputs were probed for ECadherin, $\beta$-catenin or GAPDH to demonstrate equal amounts of protein from each group were used for IPs. (G) Liver sections from mice under the HDTVI model for 4 weeks stained for V5 (green), HA (red) or myc (white) to identify nodules by immunofluorescence expressing MET, IQGAP1 and $\beta$-catenin, respectively. Tumors in B+M condition are demarcated by white dashed line. Scale bar is $50 \mu \mathrm{m}$. Graphs show mean \pm SEM and dots represent individual mice. For (E), two-way paired ANOVA with Tukey's multiple comparisons test and all others compared via Student's t-Test to determine significance. Significance is indicated with ${ }^{*} P<0.05,{ }^{* *} P<0.01$, ${ }^{* * *} P<0.001,{ }^{* * *} P<0.0001$. 

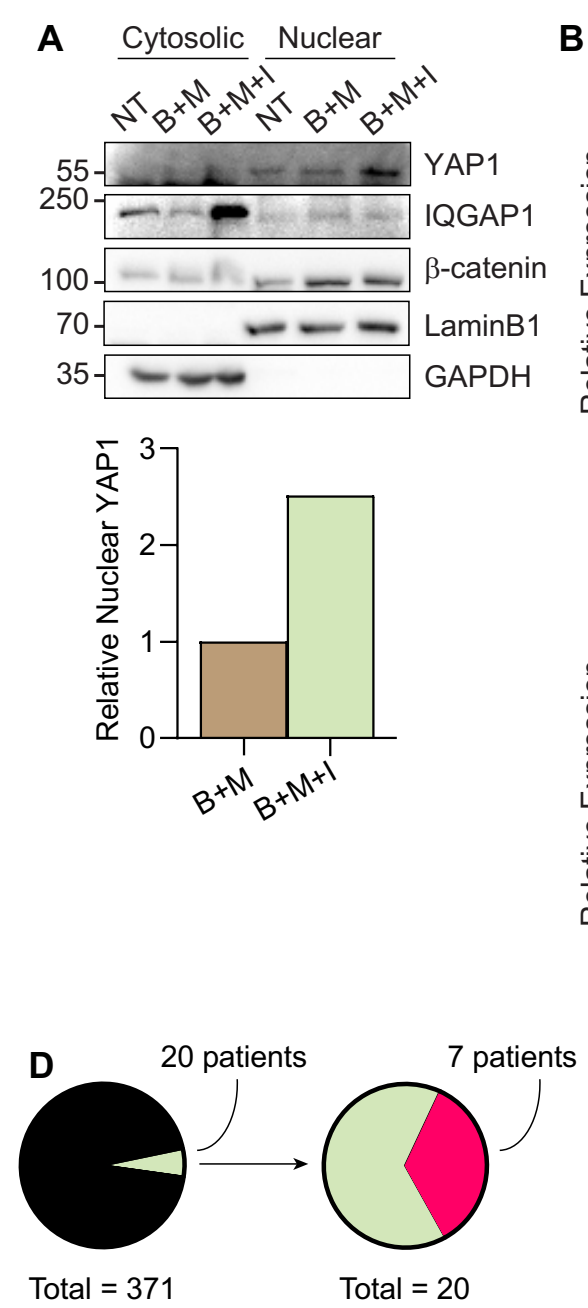

- IQGAP1 Unaltered and NUAK2 Unaltered HCC Cases OIQGAP1 ${ }^{\text {High }} \mathrm{HCC}$ Cases oNUAK2 ${ }^{\text {High }}$ HCC Cases

E

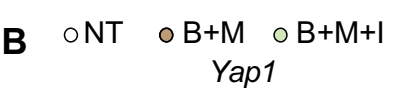
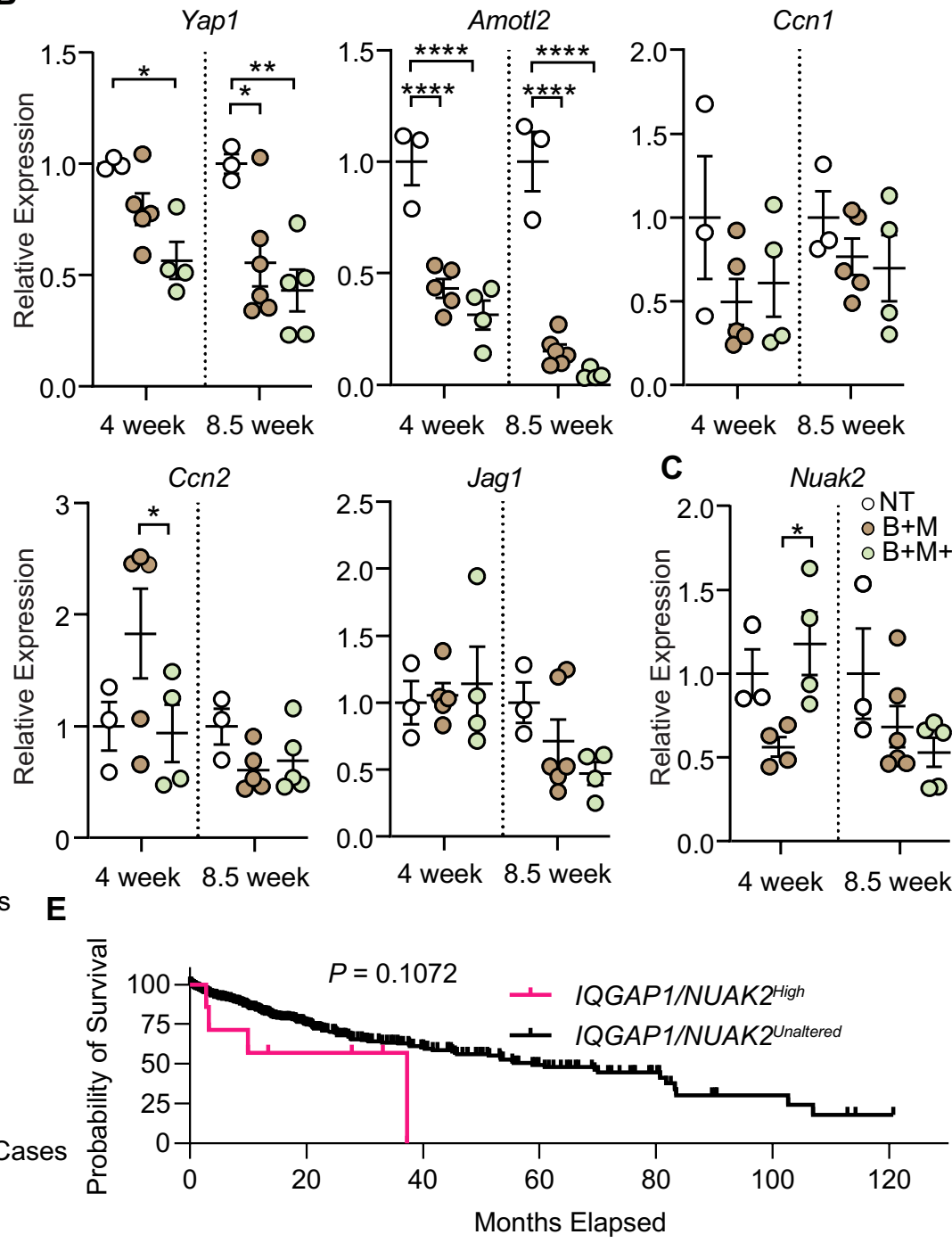

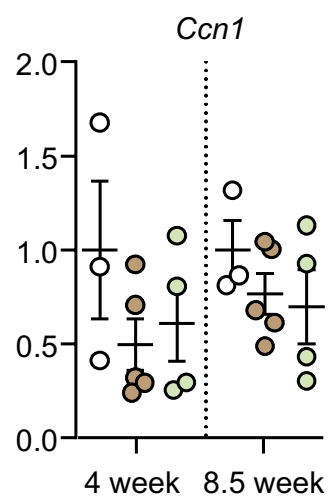

C

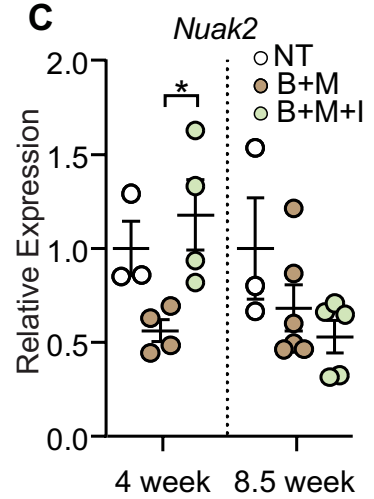

Figure 6. IQGAP1 overexpression drives HCC pathogenesis by inducing Hippo/YAP signaling in vivo. (A) Cytosolic and nuclear protein from whole livers (4 week pooled NT $n=3$, $B+M n=5$ and $B+M+I n=4$ ) were analyzed for YAP1, IQGAP1 and $\beta$-catenin. Cytosolic protein was normalized to GAPDH and nuclear protein normalized to LaminB1. GAPDH and LaminB1 show purity of cytosolic or nuclear fractions, respectively. (B) Expression of Yap1 and Hippo/YAP target genes Amotl2, Ccn1, Ccn2, and Jag1 in whole livers from 4- and 8.5-week samples, normalized to Gapdh. (C) Expression of Nuak2 in whole livers from 4 and 8.5-week $\mathrm{NT}, \mathrm{B}+\mathrm{M}$ and $\mathrm{B}+\mathrm{M}+\mathrm{I}$ samples, normalized to Gapdh. (D) Pie charts demonstrating the distribution of HCC cases with IQGAP1 $1^{\text {High }}$ expression from the TCGA cohort. Distribution further breaks down the number of cases with IQGAP1 ${ }^{\text {High }}$ that also contain NUAK2 $2^{\text {High }}$ expression. (E) Overall survival for subset of patients with IQGAP1 ${ }^{\text {High }} / N U A K 2^{\text {High }}$ expression

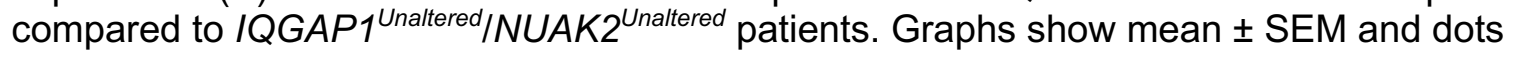
represent individual mice. For (B), two-way paired ANOVA with Tukey's multiple comparisons test and for (C) Log-rank (Mantel-Cox) test was used to determine significance for survival curve and Student's t-Test was used everywhere else. Significance is indicated with ${ }^{*} P<0.05$, ${ }^{* *} P<$ $0.01{ }^{* * * *} P<0.0001$. 
Low IQGAP1<smiles>[Tl]C1CCCCC1</smiles>

MET, AKT-1, S6 activation

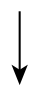

Cell proliferation and tumorigenesis

Figure 7. Summary of findings. Using two models of liver tumors, diethylnitrosamine, which produces both $\beta$-catenin and MAPK positive tumors, and a sleeping beauty hydrodynamic tail vein injection model overexpressing $\beta$-catenin and MET, we show that both overexpression and deletion of IQGAP1 promotes cell proliferation and increased tumor burden via enhanced Hippo signaling. IQGAP1 deletion results in increased MET activation and functions via AKT-1 and S6 signaling resulting in enhanced cell proliferation and tumor growth. IQGAP1 overexpression results in increased NUAK2 expression permitting increased YAP1 nuclear translocation which promotes accelerated HCC neoplasia. 


\section{References}

$1 \quad$ Llovet JM, Ricci S, Mazzaferro V, Hilgard P, Gane E, Blanc JF et al. Sorafenib in advanced hepatocellular carcinoma. The New England journal of medicine 2008; 359: 378-390.

2 Martini A, Ceranto E, Gatta A, Angeli P, Pontisso P. Occult liver disease burden: Analysis from a large general practitioners' database. United European gastroenterology journal 2017; 5: 982-986.

3 Kudo M, Finn RS, Qin S, Han KH, Ikeda K, Piscaglia F et al. Lenvatinib versus sorafenib in first-line treatment of patients with unresectable hepatocellular carcinoma: a randomised phase 3 non-inferiority trial. Lancet 2018; 391: 1163-1173.

4 Zhu AX, Rosmorduc O, Evans TR, Ross PJ, Santoro A, Carrilho FJ et al. SEARCH: a phase III, randomized, double-blind, placebo-controlled trial of sorafenib plus erlotinib in patients with advanced hepatocellular carcinoma. J Clin Oncol 2015; 33: 559-566.

5 Johnson PJ, Qin S, Park JW, Poon RT, Raoul JL, Philip PA et al. Brivanib versus sorafenib as first-line therapy in patients with unresectable, advanced hepatocellular carcinoma: results from the randomized phase III BRISK-FL study. J Clin Oncol 2013; 31: 3517-3524.

$6 \quad$ Llovet JM, Decaens T, Raoul JL, Boucher E, Kudo M, Chang C et al. Brivanib in patients with advanced hepatocellular carcinoma who were intolerant to sorafenib or for whom sorafenib failed: results from the randomized phase III BRISK-PS study. J Clin Oncol 2013; 31: 3509-3516.

7 Cheng AL, Kang YK, Lin DY, Park JW, Kudo M, Qin S et al. Sunitinib versus sorafenib in advanced hepatocellular cancer: results of a randomized phase III trial. J Clin Oncol 2013; 31: 4067-4075.

8 Cainap C, Qin S, Huang WT, Chung IJ, Pan H, Cheng Y et al. Linifanib versus Sorafenib in patients with advanced hepatocellular carcinoma: results of a randomized phase III trial. J Clin Oncol 2015; 33: 172-179.

9 Zhu AX, Kudo M, Assenat E, Cattan S, Kang YK, Lim HY et al. Effect of everolimus on survival in advanced hepatocellular carcinoma after failure of sorafenib: the EVOLVE-1 randomized clinical trial. JAMA 2014; 312: 57-67.

10 White CD, Brown MD, Sacks DB. IQGAPs in cancer: a family of scaffold proteins underlying tumorigenesis. FEBS letters 2009; 583: 1817-1824.

11 White CD, Khurana H, Gnatenko DV, Li Z, Odze RD, Sacks DB et al. IQGAP1 and IQGAP2 are reciprocally altered in hepatocellular carcinoma. BMC gastroenterology 2010; 10: 125.

12 Chen F, Zhu HH, Zhou LF, Wu SS, Wang J, Chen Z. IQGAP1 is overexpressed in hepatocellular carcinoma and promotes cell proliferation by Akt activation. Experimental \& molecular medicine 2010; 42: 477-483. 
13 Xia FD, Wang ZL, Chen HX, Huang Y, Li JD, Wang ZM et al. Differential expression of IQGAP1/2 in Hepatocellular carcinoma and its relationship with clinical outcomes. Asian Pac J Cancer Prev 2014; 15: 4951-4956.

14 Jin X, Liu Y, Liu J, Lu W, Liang Z, Zhang D et al. The Overexpression of IQGAP1 and beta-Catenin Is Associated with Tumor Progression in Hepatocellular Carcinoma In Vitro and In Vivo. PLoS One 2015; 10: e0133770.

15 Jameson KL, Mazur PK, Zehnder AM, Zhang J, Zarnegar B, Sage J et al. IQGAP1 scaffold-kinase interaction blockade selectively targets RAS-MAP kinase-driven tumors. Nature medicine 2013; 19: 626-630.

16 Liang Z, Yang Y, He Y, Yang P, Wang X, He G et al. SUMOylation of IQGAP1 promotes the development of colorectal cancer. Cancer letters 2017; 411: 90-99.

17 Schmidt VA, Chiariello CS, Capilla E, Miller F, Bahou WF. Development of hepatocellular carcinoma in Iqgap2-deficient mice is IQGAP1 dependent. Mol Cell Biol 2008; 28: 1489-1502.

18 Liu C, Billadeau DD, Abdelhakim H, Leof E, Kaibuchi K, Bernabeu C et al. IQGAP1 suppresses TbetaRII-mediated myofibroblastic activation and metastatic growth in liver. J Clin Invest 2013; 123: 1138-1156.

19 Hensel J, Duex JE, Owens C, Dancik GM, Edwards MG, Frierson HF et al. Patient Mutation Directed shRNA Screen Uncovers Novel Bladder Tumor Growth Suppressors. Mol Cancer Res 2015; 13: 1306-1315.

20 Bessede E, Molina S, Acuna-Amador L, Dubus P, Staedel C, Chambonnier L et al. Deletion of IQGAP1 promotes Helicobacter pylori-induced gastric dysplasia in mice and acquisition of cancer stem cell properties in vitro. Oncotarget 2016; 7: 80688-80699.

21 Vesselinovitch SD, Mihailovich N. Kinetics of diethylnitrosamine hepatocarcinogenesis in the infant mouse. Cancer Res 1983; 43: 4253-4259.

22 Naugler WE, Sakurai T, Kim S, Maeda S, Kim K, Elsharkawy AM et al. Gender disparity in liver cancer due to sex differences in MyD88-dependent IL-6 production. Science 2007; 317: 121-124.

23 Tolba R, Kraus T, Liedtke C, Schwarz M, Weiskirchen R. Diethylnitrosamine (DEN)induced carcinogenic liver injury in mice. Lab Anim 2015; 49: 59-69.

24 Erickson HL, Anakk S. Identification of IQ motif-containing GTPase-activating protein 1 as a regulator of long-term ketosis. JCl insight 2018; 3.

25 Zoheir KM, Abd-Rabou AA, Harisa GI, Ashour AE, Ahmad SF, Attia SM et al. Gene expression of IQGAPs and Ras families in an experimental mouse model for hepatocellular carcinoma: a mechanistic study of cancer progression. Int J Clin Exp Pathol 2015; 8: 8821-8831. 
26 Kunimoto K, Nojima H, Yamazaki Y, Yoshikawa T, Okanoue T, Tsukita S. Involvement of IQGAP3, a regulator of Ras/ERK-related cascade, in hepatocyte proliferation in mouse liver regeneration and development. J Cell Physiol 2009; 220: 621-631.

27 Shi Y, Qin N, Zhou Q, Chen Y, Huang S, Chen B et al. Role of IQGAP3 in metastasis and epithelial-mesenchymal transition in human hepatocellular carcinoma. $J$ Trans/ Med 2017; 15: 176.

28 Boyault S, Rickman DS, de Reynies A, Balabaud C, Rebouissou S, Jeannot E et al. Transcriptome classification of HCC is related to gene alterations and to new therapeutic targets. Hepatology (Baltimore, Md) 2007; 45: 42-52.

29 Calderaro J, Couchy G, Imbeaud S, Amaddeo G, Letouze E, Blanc JF et al. Histological subtypes of hepatocellular carcinoma are related to gene mutations and molecular tumour classification. Journal of hepatology 2017; 67: 727-738.

30 Dow M, Pyke RM, Tsui BY, Alexandrov LB, Nakagawa H, Taniguchi K et al. Integrative genomic analysis of mouse and human hepatocellular carcinoma. Proceedings of the National Academy of Sciences of the United States of America 2018; 115: E9879E9888.

31 Connor F, Rayner TF, Aitken SJ, Feig C, Lukk M, Santoyo-Lopez J et al. Mutational landscape of a chemically-induced mouse model of liver cancer. Journal of hepatology 2018; 69: 840-850.

32 Buchmann A, Karcier Z, Schmid B, Strathmann J, Schwarz M. Differential selection for B-raf and Ha-ras mutated liver tumors in mice with high and low susceptibility to hepatocarcinogenesis. Mutat Res 2008; 638: 66-74.

33 Aydinlik H, Nguyen TD, Moennikes O, Buchmann A, Schwarz M. Selective pressure during tumor promotion by phenobarbital leads to clonal outgrowth of beta-cateninmutated mouse liver tumors. Oncogene 2001; 20: 7812-7816.

34 Unterberger EB, Eichner J, Wrzodek C, Lempiainen H, Luisier R, Terranova R et al. Haras and beta-catenin oncoproteins orchestrate metabolic programs in mouse liver tumors. International journal of cancer 2014; 135: 1574-1585.

35 Bozkaya G, Korhan P, Cokakli M, Erdal E, Sagol O, Karademir S et al. Cooperative interaction of MUC1 with the HGF/c-Met pathway during hepatocarcinogenesis. Mol Cancer 2012; $11: 64$.

36 Monga SP, Mars WM, Pediaditakis P, Bell A, Mule K, Bowen WC et al. Hepatocyte growth factor induces Wnt-independent nuclear translocation of beta-catenin after Metbeta-catenin dissociation in hepatocytes. Cancer Res 2002; 62: 2064-2071.

37 Zeng G, Apte U, Micsenyi A, Bell A, Monga SP. Tyrosine residues 654 and 670 in betacatenin are crucial in regulation of Met-beta-catenin interactions. Exp Cell Res 2006; 312: 3620-3630.

38 Giordano S, Columbano A. Met as a therapeutic target in HCC: facts and hopes. Journal of hepatology 2014; 60: 442-452. 
39 Tao J, Xu E, Zhao Y, Singh S, Li X, Couchy G et al. Modeling a Human HCC Subset in Mice Through Co-Expression of Met and Point-Mutant beta-Catenin. Hepatology (Baltimore, Md) 2016.

40 Lee SH, Song IH, Noh R, Kang HY, Kim SB, Ko SY et al. Clinical outcomes of patients with advanced hepatocellular carcinoma treated with sorafenib: a retrospective study of routine clinical practice in multi-institutions. BMC Cancer 2015; 15: 236.

41 Peng SY, Chen WJ, Lai PL, Jeng YM, Sheu JC, Hsu HC. High alpha-fetoprotein level correlates with high stage, early recurrence and poor prognosis of hepatocellular carcinoma: significance of hepatitis virus infection, age, p53 and beta-catenin mutations. International journal of cancer 2004; 112: 44-50.

42 Imamura H, Matsuyama Y, Tanaka E, Ohkubo T, Hasegawa K, Miyagawa S et al. Risk factors contributing to early and late phase intrahepatic recurrence of hepatocellular carcinoma after hepatectomy. Journal of hepatology 2003; 38: 200-207.

43 Briggs MW, Li Z, Sacks DB. IQGAP1-mediated stimulation of transcriptional coactivation by beta-catenin is modulated by calmodulin. $J$ Biol Chem 2002; 277: 74537465.

44 Fukata M, Kuroda S, Nakagawa M, Kawajiri A, Itoh N, Shoji I et al. Cdc42 and Rac1 regulate the interaction of IQGAP1 with beta-catenin. J Biol Chem 1999; 274: 2604426050.

45 Kuroda S, Fukata M, Nakagawa M, Fujii K, Nakamura T, Ookubo T et al. Role of IQGAP1, a target of the small GTPases Cdc42 and Rac1, in regulation of E-cadherinmediated cell-cell adhesion. Science 1998; 281: 832-835.

46 Cox AG, Hwang KL, Brown KK, Evason K, Beltz S, Tsomides A et al. Yap reprograms glutamine metabolism to increase nucleotide biosynthesis and enable liver growth. Nat Cell Biol 2016; 18: 886-896.

47 Dong J, Feldmann G, Huang J, Wu S, Zhang N, Comerford SA et al. Elucidation of a universal size-control mechanism in Drosophila and mammals. Cell 2007; 130: 11201133.

48 Sayedyahossein S, Li Z, Hedman AC, Morgan CJ, Sacks DB. IQGAP1 Binds to Yesassociated Protein (YAP) and Modulates Its Transcriptional Activity. J Biol Chem 2016; 291: 19261-19273.

49 Anakk S, Bhosale M, Schmidt VA, Johnson RL, Finegold MJ, Moore DD. Bile acids activate YAP to promote liver carcinogenesis. Cell reports 2013; 5: 1060-1069.

50 Park YY, Sohn BH, Johnson RL, Kang MH, Kim SB, Shim JJ et al. Yes-associated protein 1 and transcriptional coactivator with PDZ-binding motif activate the mammalian target of rapamycin complex 1 pathway by regulating amino acid transporters in hepatocellular carcinoma. Hepatology (Baltimore, Md) 2016; 63: 159-172. 
51 Xu MZ, Chan SW, Liu AM, Wong KF, Fan ST, Chen J et al. AXL receptor kinase is a mediator of YAP-dependent oncogenic functions in hepatocellular carcinoma. Oncogene 2011; 30: 1229-1240.

52 Ahn EY, Kim JS, Kim GJ, Park YN. RASSF1A-mediated regulation of AREG via the Hippo pathway in hepatocellular carcinoma. Mol Cancer Res 2013; 11: 748-758.

53 Zhang J, Ji JY, Yu M, Overholtzer M, Smolen GA, Wang R et al. YAP-dependent induction of amphiregulin identifies a non-cell-autonomous component of the Hippo pathway. Nat Cell Biol 2009; 11: 1444-1450.

54 Zhao B, Ye X, Yu J, Li L, Li W, Li S et al. TEAD mediates YAP-dependent gene induction and growth control. Genes Dev 2008; 22: 1962-1971.

55 Camargo FD, Gokhale S, Johnnidis JB, Fu D, Bell GW, Jaenisch R et al. YAP1 increases organ size and expands undifferentiated progenitor cells. Curr Biol 2007; 17: 2054-2060.

56 Min Q, Molina L, Li J, Adebayo Michael AO, Russell JO, Preziosi ME et al. beta-Catenin and Yes-Associated Protein 1 Cooperate in Hepatoblastoma Pathogenesis. Am J Pathol 2019; 189: 1091-1104.

57 Zhang J, Liu P, Tao J, Wang P, Zhang Y, Song X et al. TEA Domain Transcription Factor 4 Is the Major Mediator of Yes-Associated Protein Oncogenic Activity in Mouse and Human Hepatoblastoma. Am J Pathol 2019; 189: 1077-1090.

58 Tao J, Calvisi DF, Ranganathan S, Cigliano A, Zhou L, Singh S et al. Activation of betacatenin and Yap1 in human hepatoblastoma and induction of hepatocarcinogenesis in mice. Gastroenterology 2014; 147: 690-701.

59 LaQuaglia MJ, Grijalva JL, Mueller KA, Perez-Atayde AR, Kim HB, Sadri-Vakili G et al. YAP Subcellular Localization and Hippo Pathway Transcriptome Analysis in Pediatric Hepatocellular Carcinoma. Sci Rep 2016; 6: 30238.

60 Yuan WC, Pepe-Mooney B, Galli GG, Dill MT, Huang HT, Hao M et al. NUAK2 is a critical YAP target in liver cancer. Nat Commun 2018; 9: 4834.

61 Gill MK, Christova T, Zhang YY, Gregorieff A, Zhang L, Narimatsu M et al. A feed forward loop enforces YAP/TAZ signaling during tumorigenesis. Nat Commun 2018; 9: 3510.

62 Perra A, Kowalik MA, Ghiso E, Ledda-Columbano GM, Di Tommaso L, Angioni MM et al. YAP activation is an early event and a potential therapeutic target in liver cancer development. Journal of hepatology 2014; 61: 1088-1096.

63 Schmidt VA. Watch the GAP: Emerging Roles for IQ Motif-Containing GTPaseActivating Proteins IQGAPs in Hepatocellular Carcinoma. Int J Hepatol 2012; 2012: 958673. 
64 Wang Y, Wang A, Wang F, Wang M, Zhu M, Ma Y et al. IQGAP1 activates Tcf signal independent of Rac1 and Cdc42 in injury and repair of bronchial epithelial cells. Experimental and molecular pathology 2008; 85: 122-128.

65 Monga SP. Role and regulation of beta-catenin signaling during physiological liver growth. Gene expression 2014; 16: 51-62.

66 Li Z, Kim SH, Higgins JM, Brenner MB, Sacks DB. IQGAP1 and calmodulin modulate Ecadherin function. J Biol Chem 1999; 274: 37885-37892.

67 Hedman AC, Smith JM, Sacks DB. The biology of IQGAP proteins: beyond the cytoskeleton. EMBO reports 2015; 16: 427-446.

68 Global Burden of Disease Cancer C, Fitzmaurice C, Abate D, Abbasi N, Abbastabar H, Abd-Allah F et al. Global, Regional, and National Cancer Incidence, Mortality, Years of Life Lost, Years Lived With Disability, and Disability-Adjusted Life-Years for 29 Cancer Groups, 1990 to 2017: A Systematic Analysis for the Global Burden of Disease Study. JAMA Oncol 2019.

69 Feng GS. Conflicting roles of molecules in hepatocarcinogenesis: paradigm or paradox. Cancer Cell 2012; 21: 150-154.

70 Kim D, Reyes-Ordonez A, Chen J. Lentivirus-Mediated RNAi in Skeletal Myogenesis. Methods in molecular biology 2019; 1889: 95-110.

71 Cerami E, Gao J, Dogrusoz U, Gross BE, Sumer SO, Aksoy BA et al. The cBio cancer genomics portal: an open platform for exploring multidimensional cancer genomics data. Cancer Discov 2012; 2: 401-404.

72 Gao J, Aksoy BA, Dogrusoz U, Dresdner G, Gross B, Sumer SO et al. Integrative analysis of complex cancer genomics and clinical profiles using the cBioPortal. Sci Signal 2013; 6: pl1.

73 Kramer A, Green J, Pollard J, Jr., Tugendreich S. Causal analysis approaches in Ingenuity Pathway Analysis. Bioinformatics 2014; 30: 523-530.

74 Guide for the Care and Use of Laboratory Animals. Washington (DC): National Academies Press (US) 2011. 